\title{
The deSUMOylase SENP2 coordinates homologous recombination and nonhomologous end joining by independent mechanisms
}

\author{
Alexander J. Garvin, Alexandra K. Walker, Ruth M. Densham, Anoop Singh Chauhan, Helen R. Stone, \\ Hannah L. Mackay, Mohammed Jamshad, Katarzyna Starowicz, Manuel Daza-Martin, \\ George E. Ronson, Alexander J. Lanz, James F. Beesley, and Joanna R. Morris \\ Birmingham Centre for Genome Biology, Institute of Cancer and Genomic Sciences, University of Birmingham, Edgbaston, \\ Birmingham B15 2TT, United Kingdom
}

SUMOylation (small ubiquitin-like modifier) in the DNA double-strand break (DSB) response regulates recruitment, activity, and clearance of repair factors. However, our understanding of a role for deSUMOylation in this process is limited. Here we identify different mechanistic roles for deSUMOylation in homologous recombination (HR) and nonhomologous end joining (NHEJ) through the investigation of the deSUMOylase SENP2. We found that regulated deSUMOylation of MDC1 prevents excessive SUMOylation and its RNF4-VCP mediated clearance from DSBs, thereby promoting NHEJ. In contrast, we show that HR is differentially sensitive to SUMO availability and SENP2 activity is needed to provide SUMO. SENP2 is amplified as part of the chromosome 3q amplification in many cancers. Increased SENP2 expression prolongs MDC1 focus retention and increases NHEJ and radioresistance. Collectively, our data reveal that deSUMOylation differentially primes cells for responding to DSBs and demonstrates the ability of SENP2 to tune DSB repair responses.

[Keywords: DNA repair; homologous recombination; MDC1; nonhomologous end joining; RNF4; SENP2; SUMO] Supplemental material is available for this article.

Received September 27, 2018; revised version accepted December 21, 2018.

The cellular response to DNA double-strand breaks (DSBs) comprises multiple steps; sensing and signaling the lesion, mediating the correct type of repair, clearing repair proteins, and reforming chromatin. The response involves a diverse set of signaling pathways and repair mechanisms coordinated by posttranslational modifications (PTMs) including phosphorylation, acetylation, ubiquitination, SUMOylation (small ubiquitin-like modifier), and others.

A major consequence of SUMOylation is the promotion of protein:protein interactions mediated by simple and short hydrophobic SUMO interaction motifs (SIMs) on proximal proteins (Song et al. 2004; Hecker et al. 2006; Psakhye and Jentsch 2012). In the yeast DNA damage response, a SUMO conjugation wave brought about by the interaction of the E3 SUMO ligase Siz2 with DNA and Mre11 results in modification of protein groups, thereby promoting SUMO-SIM interactions between members of those groups (Psakhye and Jentsch 2012; Jentsch and

Corresponding author: j.morris.3@bham.ac.uk

Article published online ahead of print. Article and publication date are online at http://www.genesdev.org/cgi/doi/10.1101/gad.321125.118. Freely available online through the Genes \& Development Open Access option.
Psakhye 2013; Chen et al. 2016). In humans, modification by SUMO E3 ligases PIAS1/4 (protein inhibitor of activated STAT) and CBX4 coordinate the repair response, driving the localization, activity, and stability of many signaling and repair proteins, such as RNF168, BRCA1, XRCC4, and Ku70 (Yurchenko et al. 2006, 2008; Galanty et al. 2009; Morris et al. 2009; Li et al. 2010; Danielsen et al. 2012; Ismail et al. 2012; Luo et al. 2012; Yin et al. 2012; Hang et al. 2014; Lamoliatte et al. 2014; Tammsalu et al. 2014). It also fosters key steps such as SUMO-BLM and SUMO-RPA70/RPA1 mediated promotion of RAD51 accumulation (Eladad et al. 2005; Ouyang et al. 2009; Dou et al. 2010; Shima et al. 2013). Many DSB repair factors are SUMOylated, but we currently lack understanding of specific roles for many of these modifications (for review, see Garvin and Morris 2017).

In the DSB repair response SUMOylation is closely integrated with ubiquitin $(\mathrm{Ub})$ signaling and the Ub-proteasome system (for review, see Morris and Garvin 2017). This involves SUMO targeted Ub ligases (STUbLs), that

(C) 2019 Garvin et al. This article, published in Genes \& Development, is available under a Creative Commons License (Attribution-NonCommercial 4.0 International), as described at http://creativecommons.org/licenses/by-nc/4.0/. 
bear tandem SIM motifs and recognize poly-SUMOylated or multimono-SUMOylated proteins and target them for ubiquitination and subsequent degradation. Human STUbLs include RNF111/Arkadia (Poulsen et al. 2013) and RING finger 4 (RNF4) (Tatham et al. 2008). Processing of SUMOylated proteins by RNF4 is part of the correct progression of DSB signaling and SUMOylation of MDC1 (mediator of damage checkpoint 1), RIF1 and BRCA1BARD1 result in their interaction with RNF4 and subsequent degradation after DNA damage (Galanty et al. 2012; Luo et al. 2012; Yin et al. 2012; Vyas et al. 2013; Kumar and Cheok 2017; Kumar et al. 2017). RNF4 may also regulate RPA residency on ssDNA (Galanty et al. 2012; Yin et al. 2012).

Enzymes with the ability to counter SUMO and Ub modifications have the potential to regulate DNA damage signaling and DNA repair. However, since many SUMOylated factors, and the SUMO machinery itself (Kumar et al. 2017) are eventually processed by STUbLs and degraded by the proteasome, it is also possible that the reversal of SUMO conjugation plays only a minor role in the response. Characterization of deubiquitinating enzymes has shown tremendous diversity and complexity in $\mathrm{Ub}$ regulation of the response (Nishi et al. 2014; Uckelmann and Sixma 2017) but the extent of deSUMOylation enzyme involvement is not known.

Here we establish two mechanisms of DSB repair regulation by the sentrin-specific protease 2, SENP2. First, we uncover a specific requirement for SENP2 in promoting early DSB signaling by protecting MDC1 from inappropriate SUMOylation and consequent RNF4-VCP (valosincontaining protein/p97) processing. We show interaction between SENP2 and MDC1 is released on damage to allow MDC1 SUMOylation required for its clearance. Second, we reveal that homologous recombination (HR) repair has a greater need for SUMO conjugates than nonhomologous enjoining (NHEJ), and thus requires SUMO proteases to contribute to the supply or redistribution of SUMO. We propose that deSUMOylation is critical to the tuning of both major DSB repair pathways.

\section{Results}

SENP2 promotes DNA damage signaling and DNA repair

In a prior siRNA screen of SUMO proteases using integrated reporters to measure HR and NHEJ we noted that siRNA to SENP2 resulted in impairment of both repair pathways (Garvin et al. 2013). To address whether SENP2 has a role in DNA repair we compared irradiation (IR)-induced $\gamma \mathrm{H} 2 \mathrm{AX}\left(\mathrm{pSer}^{139}\right.$-H2AX) focus clearance, indicative of DNA repair (Supplemental Fig. S1A), and cellular sensitivity to irradiation or camptothecin (CPT) of wild-type and SENP2 CRISPR knockout HAP1 cells (SENP2 knockout) (Supplemental Fig. S1BD). SENP2 knockout cells showed both delay in IR induced $\gamma \mathrm{H} 2 \mathrm{AX}$ focus clearance and greater sensitivity to DNA damaging agents than wild-type cells (Supplemental Fig. S1A-D).
SENP2 localizes to several subcellular compartments and is enriched at nuclear pores (Hang and Dasso 2002; Zhang et al. 2002; Panse et al. 2003; Makhnevych et al. 2007; Goeres et al. 2011; Chow et al. 2014; Tan et al. 2015; Odeh et al. 2018). We generated a siRNA-resistant SENP2 ${ }^{\text {WT }}$ catalytic mutant (C548A) and a mutant with reduced nuclear pore targeting (NPm, as described previously) (illustrated in Supplemental Fig. S1E; Goeres et al. 2011; Odeh et al. 2018). Depletion of SENP2 in HeLa resulted in radio-sensitivity that could be complemented with siRNA-resistant SENP2 ${ }^{\mathrm{WT}}$ and SENP2 ${ }^{\mathrm{NPm}}$ but not by SENP2 ${ }^{\text {C548A }}$ in colony assays (Fig. 1A; Supplemental Fig. $\mathrm{S} 1 \mathrm{~F})$. Survival in response to CPT and olaparib and measures of both HR and NHEJ repair were also dependent on the catalytic activity of SENP2 (Fig. 1B,C; Supplemental Fig. S1G). These data illustrate a need for catalytically competent SENP2 in DNA DSB repair.

SUMO1 and SUMO2/3 colocalize with $\gamma \mathrm{H} 2 \mathrm{AX}$ foci in response to genotoxic stress such as IR /Galanty et al. 2009; Morris et al. 2009); however, following IR, we observed less SUMO colocalization in siSENP2 cells (Fig. 1D; Supplemental Fig. S1H). Since a potential cause of SUMO conjugate loss at DSBs is a reduction in the recruitment of proteins on which SUMOylation occurs (Galanty et al. 2009; Morris et al. 2009), we examined cells for DSB repair factor foci. MDC1 is recruited to $\gamma \mathrm{H} 2 \mathrm{AX}$ and begins a Ub signaling cascade involving the E3 Ub ligases RNF8/ RNF168 to promote the recruitment of the BRCA1-A complex and 53BP1 complex (for review, see Panier and Boulton 2014). In siSENP2 cells MDC1 colocalization with $\gamma \mathrm{H} 2 \mathrm{AX}$ was observed shortly after IR; however, RNF8, RNF168, Ub conjugates linked through Lys63 (K63-Ub), 53BP1, and BRCA1 showed incomplete, or severely reduced, recruitment (Fig. 1E). Together these data indicate a role for SENP2 in early DSB signaling.

\section{RNF4-VCP is responsible for defective DNA damage signaling in SENP2-depleted cells}

To determine the signaling breakpoint in SENP2-deficient cells, we examined MDC1, GFP-RNF168, and 53BP1 focus kinetics following IR. Depletion of SENP2 severely reduced the accumulation of 53BP1 and RNF168 foci throughout the time course; however, MDC1 foci initially formed in siSENP2 and SENP2 knockout cells and then rapidly became undetectable (Fig. 1F; Supplemental Fig. S1I,J). The formation of both MDC1 and 53BP1 foci at later time points, $4 \mathrm{~h}$ after IR, were restored in SENP2 ${ }^{\mathrm{WT}}$ - but not SENP2 ${ }^{\mathrm{C} 548 \mathrm{~A}}$-complemented cells (Fig. 1I-K), suggesting that deSUMOylase activity is important to the persistence of $\mathrm{MDC} 1$ at sites of damage and to the accumulation of 53BP1 foci.

To address which factors are responsible for the rapid clearance of MDC1 in SENP2-deficient cells, we first investigated RNF4, whose activity has been implicated in MDC1 turnover (Galanty et al. 2012; Luo et al. 2012; Yin et al. 2012; Hendriks and Vertegaal 2015; Hendriks et al. 2015). Codepletion of RNF4 with SENP2 resulted in focus kinetics of MDC1, RNF168, and 53BP1 similar to that of control-treated cells (Fig. 1F-H). The pattern of 
A

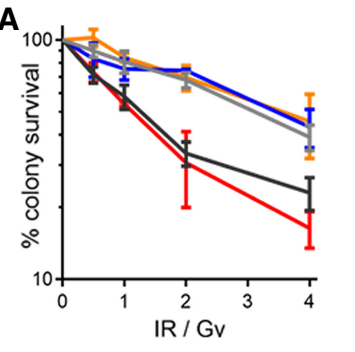

D

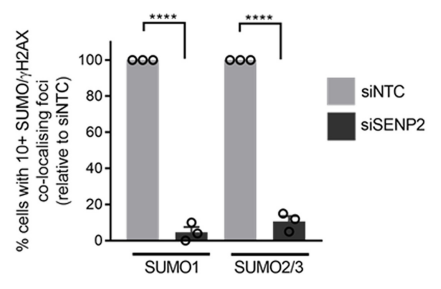

- sinTC

- siSENP2

- SISENP2 + WT SENP2

- siSENP2 + C548A SENP2

- siSENP2 + NPm SENP2
B

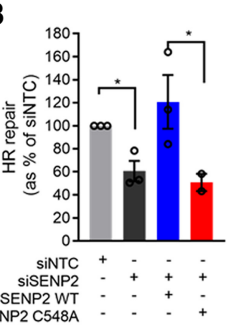

C

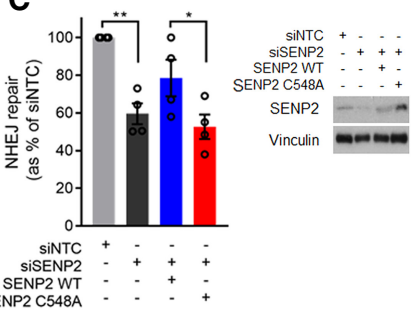

E

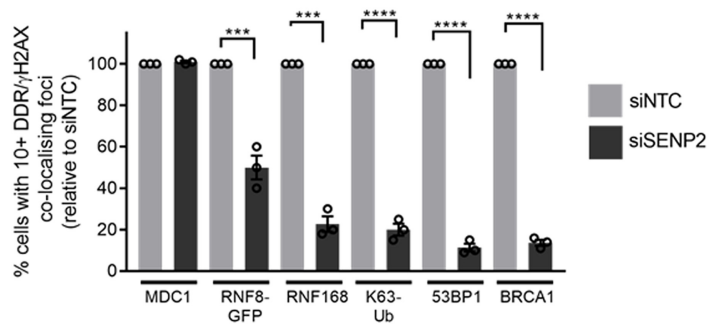

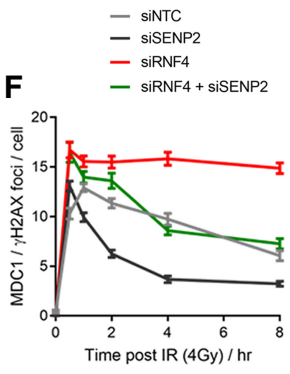

G

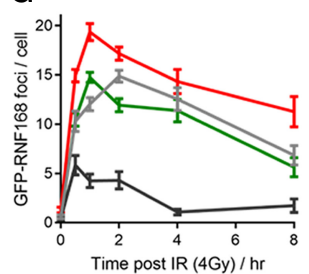

J

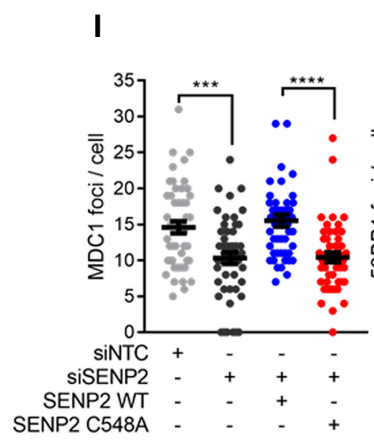

$\mathbf{L}$
H

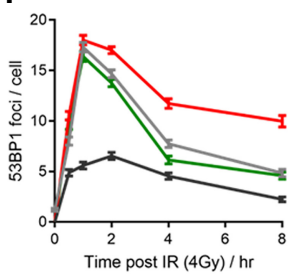

53BP1 (4hr post IR) siNTC siRNF4

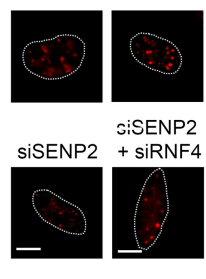

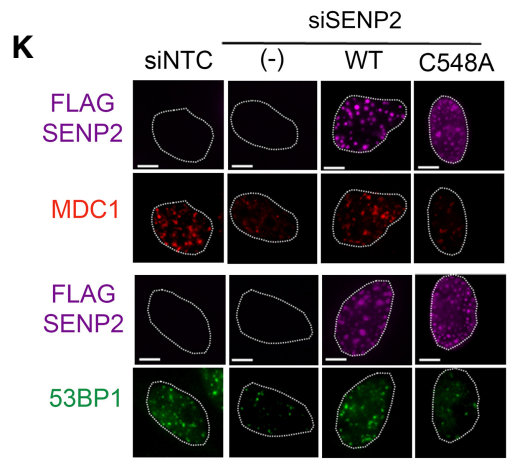

M

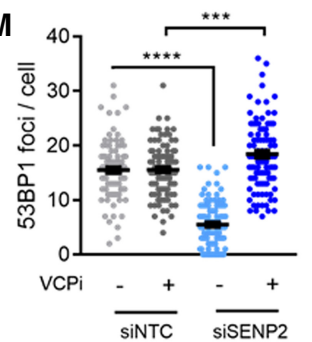

Figure 1. SENP2 promotes DNA damage signaling and DNA repair. $(A)$ IR colony survival in HeLa treated with siNTC or siSENP2 for $72 \mathrm{~h}$. Cells were treated concurrently with $1 \mu \mathrm{g} / \mathrm{mL}$ doxycycline to induce siRNA-resistant forms of SENP2. $n=4$. (B,C) HR (U2OS DR3GFP) or NHEJ (U2OS-EJ5-GFP) assays using siSENP2 or siNTC treated cells transfected with RFP, I-SceI, and SENP2 ${ }^{\text {WT }}$ or SENP2 ${ }^{\mathrm{C} 548 \mathrm{~A}}$. $\mathrm{GFP}^{+}$cells were normalized to RFP transfection efficiency. Percentage repair is given compared with siNTC. Western blot shows SENP2 knockdown efficiency and restoration with siRNA resistant cDNA. $n=3$. $(D, E)$ SUMO $/ \gamma \mathrm{H} 2 \mathrm{AX}$ colocalizing foci in HeLa siNTC or siSENP2 cells fixed $1 \mathrm{~h}$ after 5 Gy of IR. (E) As for $D$ with the indicated DDR factors. $n=3$. (F-H) Time course of MDC1 ( $n=200$ ), GFPRNF168 ( $n=50)$, or 53BP1 $(n=150)$ foci in HeLa treated with indicated siRNA for $72 \mathrm{~h}$. Representative images for 53BP1 foci at $4 \mathrm{~h}$ after IR are shown. $(I-K) \mathrm{MDC} 1$ and 53BP1 foci per cell, respectively, $4 \mathrm{~h}$ after $4 \mathrm{~Gy}$ of IR in siNTC or siSENP2 HeLa. $(K)$ representative images related to $I, J, n=100$ cells. $(L, M)$ HeLa (siNTC/siSENP2) irradiated with 4 Gy and, $0.5 \mathrm{~h}$ later, treated with DMSO/0.1 $\mu M$ VCPi, CB-5083. Cells were fixed at the indicated times and scored for MDC1 foci. $(M)$ As for $L$ but 53BP1 foci in cells fixed at $2 \mathrm{~h}$. $n=100$ cells. 
total SUMO conjugates seen following IR suggested a similar relationship between SENP2 and RNF4. Control cells exhibited a global increase in high-molecular-weight SUMO conjugates, particularly for SUMO2/3, after treatment (Supplemental Fig. S2A,B). Whereas in siSENP2 cells, SUMO conjugates were constitutively higher in untreated cells and showed only a slight increase after IR (Supplemental Fig. S2A,B), consistent with the observation of poor DDR protein recruitment and SUMO IRIF formation. Conjugate patterns after siRNF4+siSENP2 codepletion resembled those seen in siNTC cells (Supplemental Fig. S2A,B) consistent with the near normal DDR focus kinetics observed on codepletion. Intriguingly, loss of the closely related protease SENP1 did not have a similar impact on SUMO conjugates, and depleted cells showed an exaggerated induction of SUMO conjugates following IR (Supplemental Fig. S2C).

RNF4-dependent substrate ubiquitination is frequently followed by processing through VCP hexameric AAA ATPase (Dantuma et al. 2014; Torrecilla et al. 2017). We compared the effects of proteasome (MG132) or VCP inhibition (CB-5083) on MDC1 focus loss after IR. As proteasome inhibition depletes the free $\mathrm{Ub}$ pool, in turn causing a failure in Ub signaling in DSB repair (Butler et al. 2012), we also transfected the cells with myc-Ub. MG132 treatment resulted in increased MDC1 focus retention, but, in cells expressing additional myc-Ub, focus numbers were reduced, suggesting that $\mathrm{Ub}$ rather than the proteasome is critical to MDC1 focus clearance (Supplemental Fig. S2D,E). In contrast, MDC1 focus persistence in the presence of VCP inhibition was unaffected by Ub expression (Supplemental Fig. S2D,E). Moreover, in SENP2-depleted cells the addition of CB-5083 restored near-normal MDC1 focus kinetics and the ability to support downstream 53BP1 foci (Fig. 1L,M). Thus, RNF4-VCP contributes to the rapid MDC1 focus kinetics in SENP2deficient cells.

In a further test for potential nuclear pore involvement we examined cells depleted for nuclear pore subcomplex components and known SENP2-interacting proteins; NUP153 and NUP107 (Goeres et al. 2011). Reduction in NUP107 had no effect on MDC1 kinetics, and NUP153 depletion modestly increased focus clearance (Supplemental Fig. S2F), confirming no substantial involvement of the nuclear pore in MDC1 kinetics. In contrast, when we codepleted the ligase responsible for MDC1 SUMOylation, PIAS4 (Luo et al. 2012), we found that siPIAS4 (but not siPIAS1) slowed MDC1 focus clearance in siSENP2 cells (Supplemental Fig. S2G). These data consolidate the notion that SUMOylation contributes to the rapid loss of MDC1 foci observed on SENP2 loss.

\section{MDC1 is a SENP2 substrate and hypo-SUMOylation of MDC1 permits DDR signaling}

Lysine 1840 is the main SUMO acceptor site on MDC1 (Supplemental Fig. S3A; Luo et al. 2012). To test whether MDC1 might be a substrate of SENP2 we generated cells expressing myc-MDC $1^{\mathrm{WT}}$ or $\mathrm{MDC}^{\mathrm{K} 1840 \mathrm{R}}$ and assayed focus kinetics in SENP2-depleted cells. MDC1 ${ }^{\mathrm{WT}}$ underwent accelerated clearance in siSENP2 cells, as observed for endogenous MDC1. However, MDC1 $1^{\text {K1840R }}$ was resistant to the effects of siSENP2, showing the same increased focus retention (as reported earlier by Luo et al. 2012) in control and siSENP2 cells (Fig. 2A, see MDC1 and $\mathrm{MDC} 1^{\mathrm{K} 1840 \mathrm{R}}$ expression data in Fig. 4D, below). Furthermore, expression of this mutant also permitted the formation of downstream 53BP1 foci in siSENP2-treated cells (Fig. 2A,B; Supplemental Fig. S3B). Since loss of the main MDC1 SUMOylation site renders damage signaling resistant to the effects of SENP2 repression, these data suggest that the impact of SENP2 loss occurs through modification at K1840-MDC1.

We purified $\mathrm{His}_{6}$-SUMO1 and His ${ }_{6}$-SUMO2 from untreated and IR-treated cells (harvested $1 \mathrm{~h}$ after IR to capture the MDC1 clearance phase) to test the impact of SENP2 on MDC1-SUMOylation. In untreated siSENP2 or SENP2 knockout cells, we observed an enrichment of MDC1 in SUMO2 conjugates (Fig. 2C,D; Supplemental Fig. S3C,D). Following exposure to IR, cells with SENP2 deficiency exhibited a reduction in SUMOylated MDC1, whereas control cells showed an increase in SUMOylated MDC1 (Fig. 2C,D; Supplemental Fig. S3C,D). In siRNF4 + siSENP2-codepleted cells, the IR-dependent reduction of MDC1-SUMO2, seen in siSENP2 cells, was not observed, and instead increased MDC1-SUMO2 was evident as in control cells (Fig. 2C; Supplemental Fig. S3C,D). We also confirmed directly that SENP2 could deSUMO2ylate a fragment of MDC1 encompassing K1840 in vitro using recombinant SENP2 catalytic domain. (Supplemental Fig. S3E).

\section{A conserved coiled-coil region of SENP2 contributes to $M D C 1$ regulation}

We next assessed whether SENP2 and MDC1 interact in cells. Using Flag-RFP-SENP2 ${ }^{\mathrm{WT}}$, we were able to detect coimmunoprecipitation with endogenous MDC1 in untreated cells. The interaction was decreased within 30 min of IR (4 Gy) and did not fully return up to $5 \mathrm{~h}$ after (Fig. 2E,F). The decrease in interaction was dependent on ATM as the inhibitor KU55933 when added prior to irradiation prevented dissociation (Fig. 2E,F). In a search for regions of SENP2 that may contribute to regulation of MDC1-SUMO, we noted a conserved coiled-coil (CC) domain (Supplemental Fig. S4A,B). We generated a 28-amino-acid deletion mutant $(\triangle \mathrm{CC})$ domain and found no changes in protein localization or activity (Supplemental Fig. S4C-F). However, unlike SENP2 ${ }^{\mathrm{WT}}$, this mutant retained interaction with MDC1 after exposure to IR (Fig. $2 \mathrm{G})$. In complementation assays, SENP2 ${ }^{\mathrm{WT}}$ permitted increased MDC1 SUMO-2ylation after IR, but cells expressing SENP2 ${ }^{\triangle \mathrm{CC}}$ failed to increase MDC1 SUMOylation (Fig. $2 \mathrm{H})$. Moreover, cells complemented with SENP2 ${ }^{\triangle \mathrm{CC}}$ failed to clear MDC1 foci and were radiosensitive (Fig. 2I-K). These data suggest that dissociation of SENP2 from MDC1 requires the activity of ATM and the SENP2 CC domain and that dissociation is essential for the IRdependent SUMOylation of MDC1, focus resolution, and proper IR repair. 
A

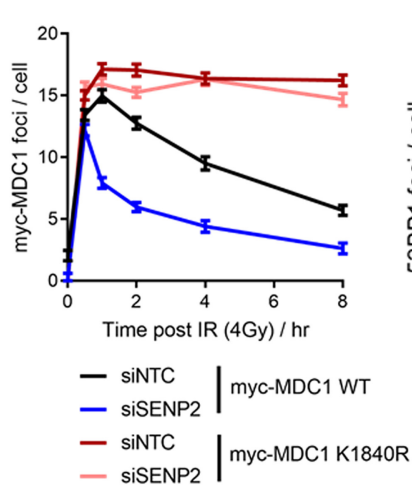

B

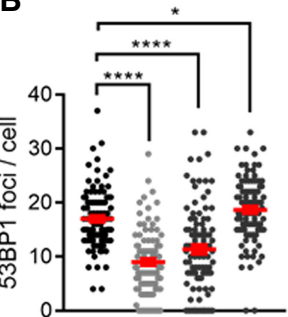

(-) WT K1840R $\overline{\text { siNTC }} \frac{\text { MDC1 }}{\text { siSENP2 }}$
C

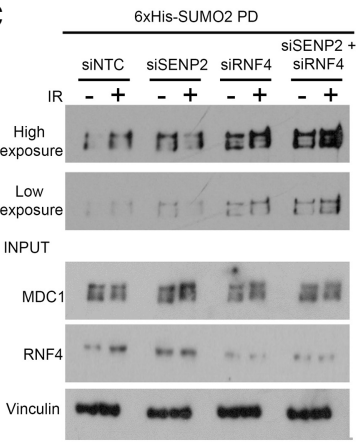

E

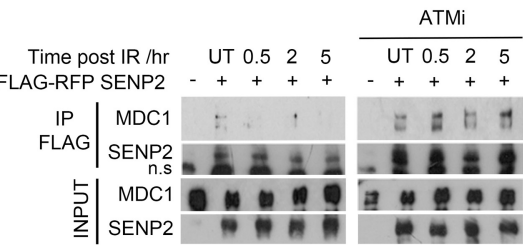

F

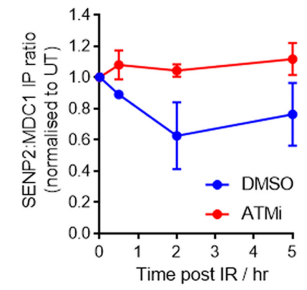

I
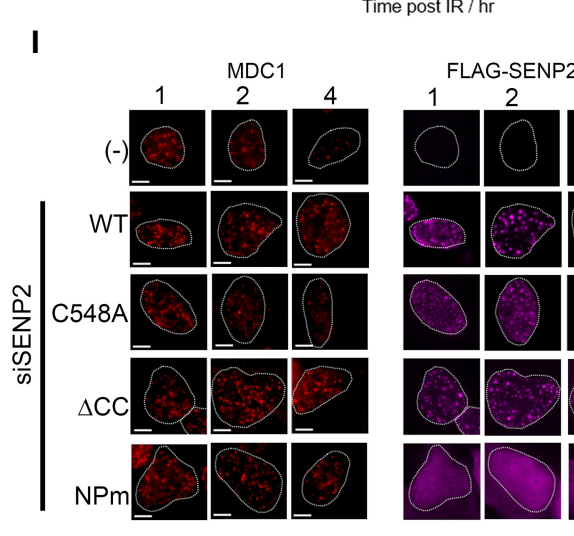

G

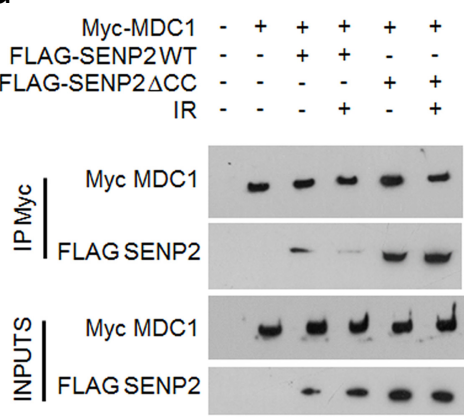

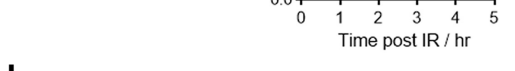

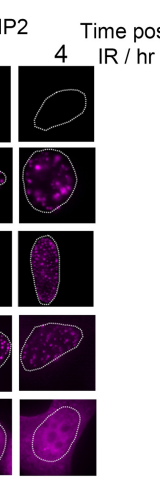

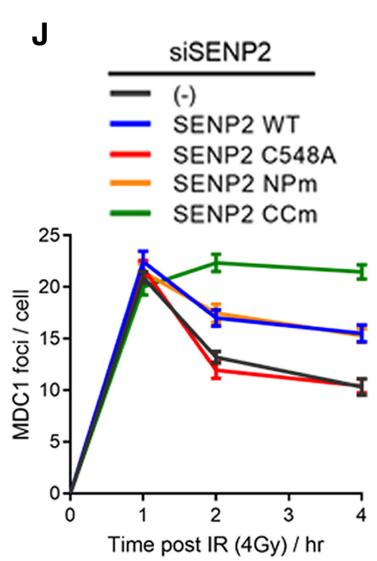

D

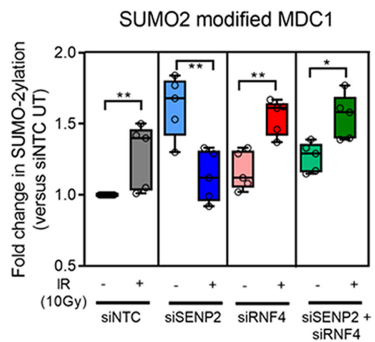

H

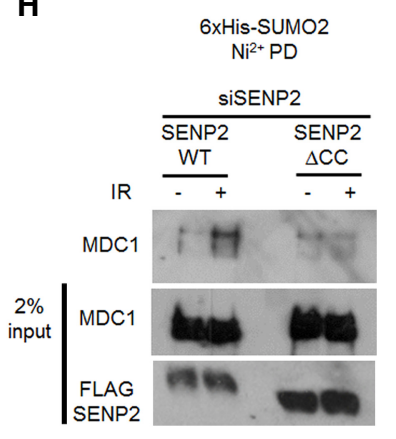

$\mathbf{K}$

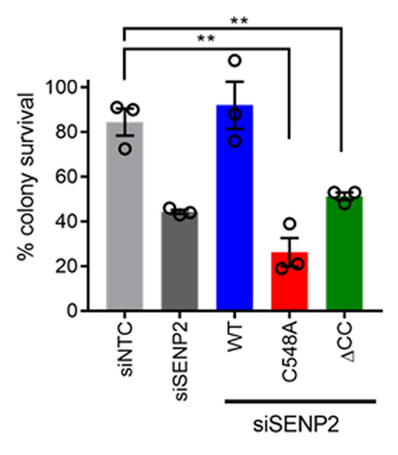

Figure 2. MDC1 is a SENP2 substrate and hypo-SUMOylation of MDC1 permits DDR signaling. $(A, B)$ HeLa treated with siRNA and induced with doxycycline for $72 \mathrm{~h}$ to express wild-type or K1840R myc-MDC1. Data show kinetics of foci per cell for the indicated times after treatment with 4 Gy of IR. (B) As for $A$ but 53BP1 foci at 2 h. $n=100$. (C). HEK293 ${ }^{\text {FlpIn }} 6 x$-His-myc SUMO2 cells treated with the indicated siRNA $(48 \mathrm{~h})$, irradiated (10 Gy), lysed $1 \mathrm{~h}$ later, and subjected to $\mathrm{Ni}^{2+}$ agarose purification followed by immunoblotting with MDC1 antibodies to determine the relative enrichment in SUMO2 conjugates. (PD) Pull-downs. (D) Quantification of the MDC1 purified by $\mathrm{Ni}^{2+}$ pull-downs in HEK293 ${ }^{\mathrm{FlpIn}}$ 6xHis-myc-SUMO2 cells illustrated in Figure 2C. Relative enrichment in SUMO2-MDC1 conjugates was determined by densitometry with the untreated siNTC sample being set as $1 . n=5$. Error bars show SEM. $(E)$ HEK293 transiently transfected with Flag-RFP-SENP2 ${ }^{\mathrm{WT}}$ for $48 \mathrm{~h}$. Thirty minutes preirradiation (4 Gy), cells were treated with $10 \mu \mathrm{MM}$ ATMi or DMSO. Lysates were made at the indicated times and immunoprecipitated with Flag agarose and blotted for endogenous MDC1 and Flag-RFP-SENP2. $(F)$ Quantification of relative Flag SENP2-MDC1 interaction ratio by densitometry, the ratio was set at 1 for untreated cells. $n=5$. Error bars represent SEM. $(G)$ HEK293 $3^{\text {FlpIn }}$ myc-MDC1 ${ }^{\text {WT }}$ transiently transfected with Flag-SENP2 ${ }^{\mathrm{WT}}$ or SENP2 ${ }^{\Delta \mathrm{CC}}$ and treated with doxycycline for $72 \mathrm{~h}$. Cells were irradiated (4 Gy) and lysed $1 \mathrm{~h}$ later followed by immunoprecipitation with myc-agarose. $(H)$ HEK293 ${ }^{\text {FlpIn }} 6 \mathrm{x}-$ His-myc SUMO2 cells, siRNA depleted for SENP2 and 24-h later transfected with SENP2 ${ }^{\mathrm{WT}}$ or SENP2 ${ }^{\triangle \mathrm{CC}}$ for an additional $48 \mathrm{~h}$. Cell lysis and $\mathrm{Ni}^{2+}$ pull-down was carried out as for $C$. $(I, J)$. HeLa treated with siSENP2. Twenty-four hours later cells were transfected with FlagSENP2 for $48 \mathrm{~h}$, irradiated (4 Gy), and fixed at indicated times. (G) MDC1 foci per cell were measured in cells costaining with Flag-SENP2. $n=50$. $(K)$ Colony survival in IR (2 Gy) HeLa treated with siNTC, siSENP2, or siSENP2 plus doxycycline to induce expression of SENP2 mutants. $n=3$. 
Requirement for SENP2 can be bypassed by increased K63-Ub signaling

We observed an initial association of MDC1 at DSBs in siSENP2 cells (Fig. 1F; Supplemental Fig. S1I,J), leading to the question of which element of the DDR is effected by rapid loss of MDC1 from damage sites. Intriguingly, a similar impact is seen on DSB signaling when MDC1 turnover is increased, but steady-state foci are only slightly altered following loss of the DUB Ataxin-3 (ATXN3) (Pfeiffer et al. 2017). We note that both SENP2 and ATXN3 contribute to the longevity of MDC1 foci and colony survival in response to IR (Supplemental Fig. S5A,B), so that together these observations suggest that MDC1 residency, or quality of MDC1 at sites of damage may promote downstream signaling. MDC1 is involved in two positive feedback loops that may require its prolonged association. It contributes to signal amplification of $\gamma \mathrm{H} 2 \mathrm{AX}$ around DNA break sites with MRN and ATM (Stucki et al. 2005; Chapman and Jackson 2008; Savic et al. 2009) and the amplification of K63-Ub linkages on Histone $\mathrm{H} 1$ and L3MBTL2 (Thorslund et al. 2015; Nowsheen et al. 2018) with RNF8 and, downstream, RNF168 (for review, see Panier and Boulton 2014). Since we observed no loss of $\gamma \mathrm{H} 2 \mathrm{AX}$ foci in SENP2-deficient cells (Supplemental Fig. S1A,I,J), we tested whether insufficient K63-Ub generation contributes to DDR signal failure by manipulating the K63-Ub machinery. Overexpression of RNF8, which catalyzes the initial K63-Ub contribution (Lok et al. 2011; Thorslund et al. 2015), and the depletion of the K63-Ub-specific Ub protease BRCC36 (depletion of which increases K63-Ub at sites of damage) (Shao et al. 2009) were capable of restoring 53BP1 foci in siSENP2 cells (Supplemental Fig. S5C-E). These data suggest that normal turnover kinetics of MDC1 at damage sites is needed for sufficient Ub conjugate generation.

\section{RNF4-VCP is responsible for the IR sensitivity of SENP2-depleted cells}

Prompted by our findings that RNF4 is responsible for rapid MDC1 focus kinetics in siSENP2-depleted cells, we next assessed whether RNF4 contributes to their radiosensitivity. Depletion of RNF4 or SENP2 individually increased cell sensitivity to IR, but codepletion resulted in IR resistance similar to siNTC cells (Fig. 3A). Expression of RNF4 ${ }^{\mathrm{WT}}$ restored resistance to RNF4-depleted cells, however, critically, on a siSENP2 + siRNF4 background reintroduction of $\mathrm{RNF} 4^{\mathrm{WT}}$ resulted in IR sensitivity (Fig. 3B), demonstrating the toxicity of RNF4 in siSENP2 cells. Complementation with RNF4 proteins that reduce interaction with its Ub loaded E2 conjugating enzyme, prevent RNF4 dimerization or interaction with SUMO (Plechanovova et al. 2012; Kung et al. 2014; Rojas-Fernandez et al. 2014) allowed survival on siRNF4 + siSENP2 backgrounds but not cells treated with siRNF4 alone (Fig. 3B). We confirmed the corollary of these findings; that SENP2 protease activity contributes to the toxicity of IR in siRNF4 cells (Fig. 3C). Moreover VCP inhibition restored IR resistance to siSENP2 cells (Fig. 3D). Thus the STUbL function of RNF4 and VCP activity contributes to the IR sensitivity of SENP2 depleted cells. Among the SENP family of SUMO proteases SENP2 is alone in contributing significantly to the lethality of IR in RNF4-depleted cells (Supplemental Fig. S5F). Together, our data reveal a strong reciprocal relationship between RNF4 and SENP2 in the cellular response to IR,
A
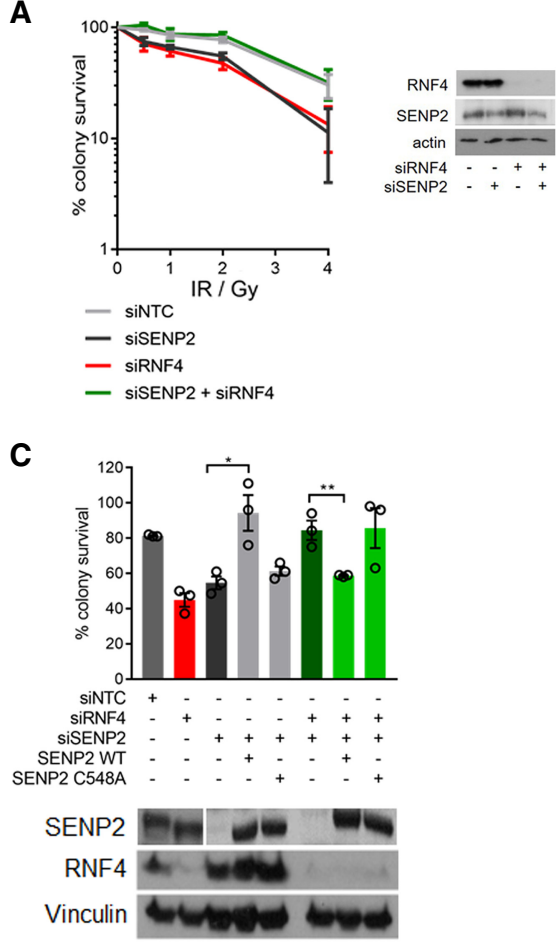

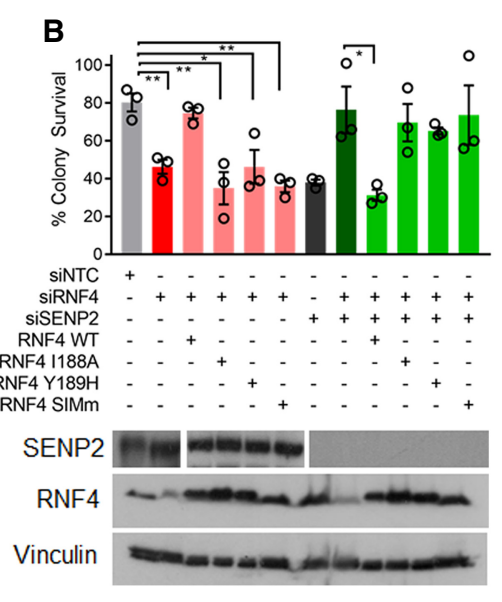

D

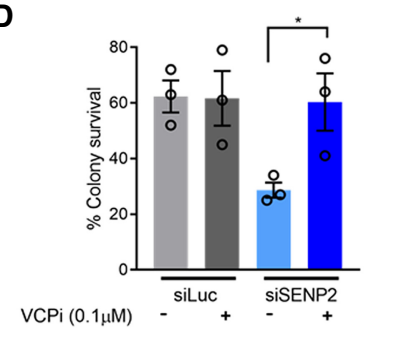

Figure 3. RNF4-VCP is responsible for the IR sensitivity of SENP2-depleted cells. (A) IR colony survival in HeLa treated with the indicated siRNA for $72 \mathrm{~h}$. (Right panel) Western blot of siRNA depletions. (B) Colony survival in HeLa ${ }^{\text {FlpIn }}$ RNF4 treated with the indicated siRNA and doxycycline to induce expression of RNF4 and its mutants for $72 \mathrm{~h}$ prior to 2 Gy IR, $n=3$. Western blot is shown below. Note that RNF4 antibody will also detect exogenous protein. $(C)$ Colony survival in $\mathrm{HeLa}^{\text {FlpIn }}$ SENP2 treated with indicated siRNA and doxycycline to induce expression of SENP2 and its mutants for $72 \mathrm{~h}$ prior to 2 Gy IR. $n=3$. Western blot is shown below. $(D)$ Colony survival of HeLa treated with the indicated siRNAs for $72 \mathrm{~h}$ and 4 Gy IR. Thirty minutes after irradiation, cells were treated with DMSO and $0.1 \mu \mathrm{M}$ VCP inhibitor CB-5083 for an additional $90 \mathrm{~min}$ prior to plating for colonies in fresh media $n=3$. 
consistent with their opposing influences on MDC1 in DSB damage signaling.

\section{SENP2 is not relevant to $S$-phase clearance of $M D C 1$}

We expected to see a role for SENP2 in regulating MDC1 at repair foci throughout the cell cycle. However, when we labeled cells with a nucleotide analog to differentiate S-phase cells, we found that SENP2 depletion had no influence on MDC1 in S-phase marked cells (Fig. 4A-C). Expression of the MDC1 $1^{\mathrm{K} 1840 \mathrm{R}}$ SUMO site mutant results in cellular IR sensitivity due to a failure of the mutant to clear from sites of DNA damage (Luo et al. 2012). We confirmed these data (Fig. 4D,E), but also challenged cells with olaparib and $\mathrm{CPT}$, agents that require $\mathrm{HR}$ repair for resistance, and found that $\mathrm{MDC1}^{\mathrm{K} 1840 \mathrm{R}}$ did not increase sensitivity to these agents (Fig. 4F,G). Moreover, the $\mathrm{MDC1} 1^{\mathrm{K} 1840 \mathrm{R}}$ mutant had no negative impact on RAD51 focus formation in S-phase cells (Fig. 4H). While $\mathrm{MDC}^{\mathrm{K} 1840 \mathrm{R}}$ expression increased 53BP1 focus numbers in $\mathrm{EdU}^{-}$cells, as reported previously (Luo et al. 2012), it did not alter 53BP1 focus number in $\mathrm{EdU}^{+}$cells (Fig. 4I). Moreover, unlike the response to IR, codepletion of RNF4 and SENP2 did not improve survival of cells challenged by olaparib or CPT and did not substantially improve HR reporter activity or RAD51 focus accumulations over single depletions (Fig. 4J-N). We conclude that $\mathrm{S}$-phase cells turn over MDC1 from broken DNA ends independently of the major MDC1 SUMO acceptor site and of SENP2, suggesting that the role of SENP2 in HR repair occurs in another pathway.

\section{$H R$ is highly sensitive to the supply of SUMO}

Since we observed increased high molecular weight SUMO conjugates in untreated cells depleted of SENP2 (Supplemental Fig. S2A) we speculated that SENP2 loss may disable HR through reduced availability of SUMO for conjugation. We overexpressed conjugation proficient and deficient SUMO in siSENP2 cells and examined survival in response to IR, CPT, or olaparib. We also assessed MDC1 foci $4 \mathrm{~h}$ after IR and RAD51 foci in S-phase cells. SUMO supplementation had no influence on IR resistance nor on MDC1 foci (Fig. 5A-C), but conjugation-competent SUMO2 improved CPT and olaparib resistance and restored RAD51 foci in SENP2-depleted cells (Fig. 5D-F). Intriguingly SENP2 depletion had no impact on RPA focus accrual, suggesting a role for SENP2 in RAD51 loading but not DNA end resection (Supplemental Fig. S5G). To test the hypothesis that differential requirements for SUMO availability exist between different repair mechanisms we performed a partial depletion of SUMO2/3 (Fig. 5G,H). Remarkably, partial SUMO2/3 depletion resulted in CPT and olaparib, but not IR, sensitivity and impaired HR, but not NHEJ in integrated repair assays (Fig. 5I,J), indicating that the $\mathrm{HR}$ pathway is more sensitive to SUMO availability. We further explored this in HAP1 cells (which, unlike HeLa, have an abundant pool of free SUMO) that had been enriched in G1, S, or M phases of the cell cycle. In G1 there was no difference in free SUMO2/3 levels between wild-type and SENP2 knockout cells, while in S- and M-phase enriched cells we noted lower levels of free SUMO in SENP2 knockout cells versus wild-type cells (Fig. 5K). Therefore SENP2 is important for maintaining a free SUMO pool during stages of the cell cycle in which HR occurs. Many HR factors are known to be SUMO modified (Garvin and Morris 2017). Increasing available SUMO circumvents the loss of SENP2 in HR sensitivity; therefore, we could not use the $6 \times$ histidineSUMO pull-down assays used previously to detect MDC1 SUMOylation. Therefore, to measure endogenous SUMO association of HR factors, we used immobilized SIM peptides to enrich SUMO-associated factors from lysates derived from control or CPT-treated HAP1 cells. We confirmed that the SIM peptide was enriching SUMO2/3 by using mutant peptides lacking a functional SIM (Supplemental Fig. S5H). We probed these pull-downs against a number of HR factors and detected increased SUMO association following CPT treatment with BLM, SMARCAD1, KAP1, and RPA70 antibodies in wild-type HAP1 cells. However, in SENP2 knockout cells, the CPT-dependent increase in SUMO association was seen with KAP1 and RPA70 but not with BLM and SMARCAD1 (Supplemental Fig. S5H). While we tested only a small panel of HR factors, these results suggest that CPT-dependent changes in SUMOylation/SUMO association are disrupted in SENP2-deficient cells in at least two HR factors. Intriguingly, BLM SUMOylation has been shown to be important for RAD51 loading (Ouyang et al. 2009), a step that is deficient in SENP2 disrupted cells (Fig. 4M).

\section{High levels of SENP2 disrupt DSB repair}

The SENP2 gene maps to chromosome 3q26-29, a region commonly amplified in epithelial cancers of the lung, ovaries, esophagus, and head and neck (Supplemental Fig. S6A,B; Qian and Massion 2008; Cancer Genome Atlas Network 2015). In lung cancer, high SENP2 mRNA levels correlate both with copy number and reduced patient survival (Supplemental Fig. S6C,D).

The $3 \mathrm{q}$ amplification carries two more genes involved in DNA repair: the Ub ligase RNF168, and the deubiquitinating enzyme USP13 (Supplemental Fig. S6E), which contribute to DNA-damage signaling (Doil et al. 2009; Stewart et al. 2009; Nishi et al. 2014; Chroma et al. 2017; Li et al. 2017). We compared the influence of high expression of each repair gene in $\mathrm{HeLa}^{\mathrm{FlpIn}}$ stable doxycycline-inducible cells. Of the three genes, SENP2 had the greatest influence on IR resistance, while increased SENP2 and USP13 reduced CPT resistance (Supplemental Fig. S6F-H)

Since our data show a critical role for SENP2 in DSB repair, we explored whether increased SENP2 expression alters repair. We found that elevation of SENP2 expression resulted in increased 53BP1 and MDC1-dependent resistance to IR (Fig. 6A,B) and was accompanied by persistent MDC1 foci at $2 \mathrm{~h}$ after IR (Fig. 6C,D). With the exception of SENP6, no other SENP expression slowed MDC1 clearance (Supplemental Fig. S6I,J). High expression of SENP2 also induced a 2.5-fold increase in NHEJ measured from an 
A

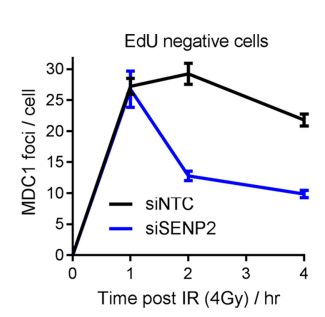

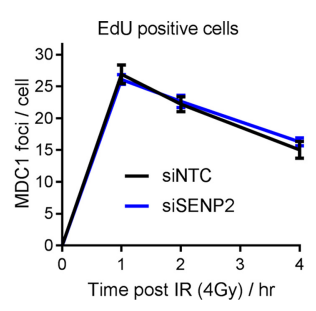
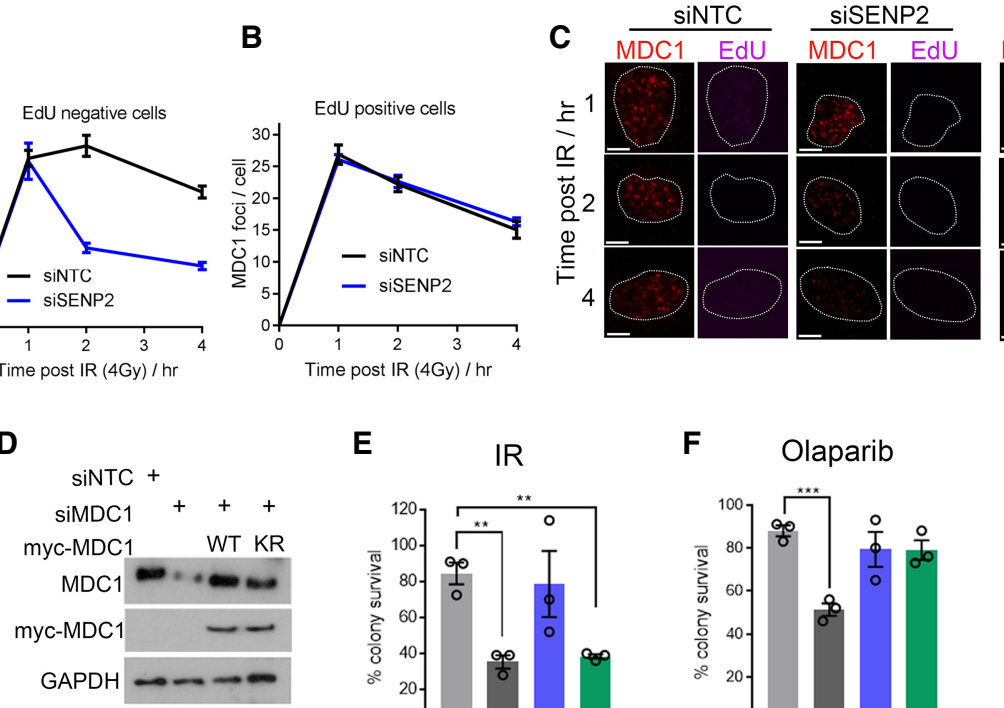

E

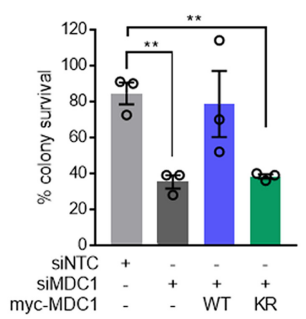

$\mathbf{F}$

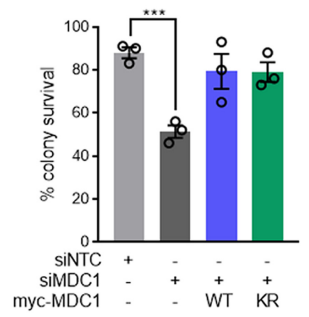

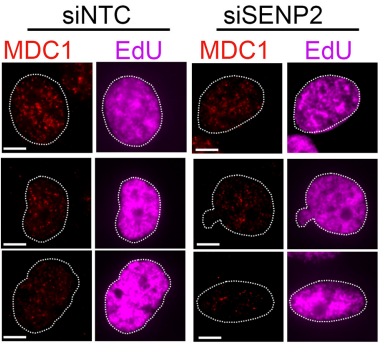

G Camptothecin

H

I $53 \mathrm{BP} 1$
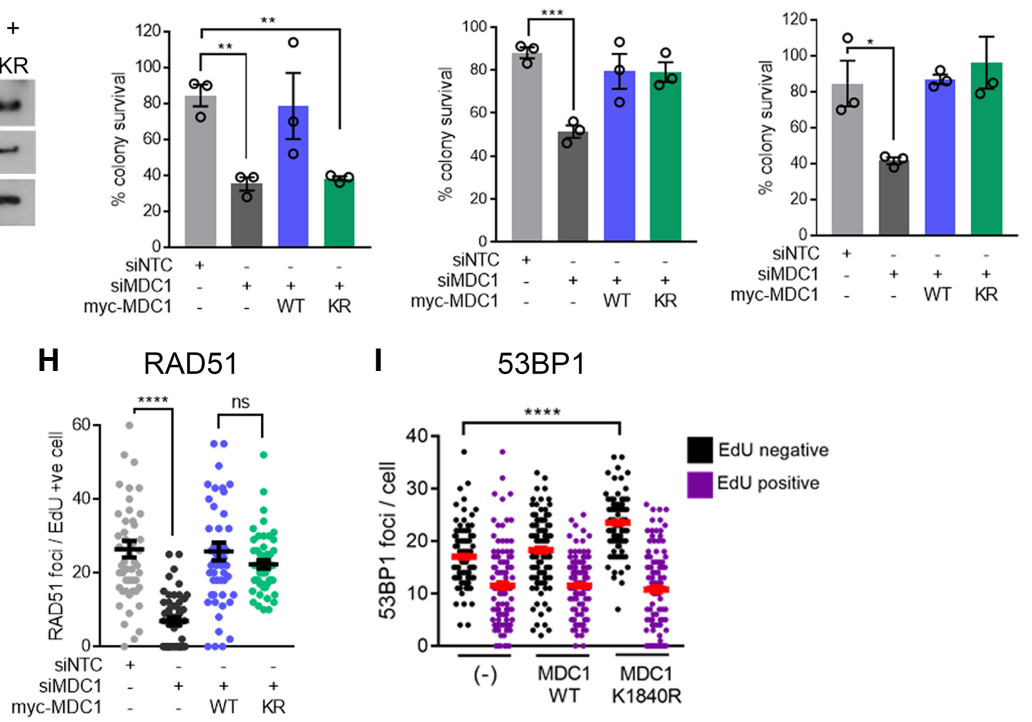

$\mathbf{J}$

\section{K}

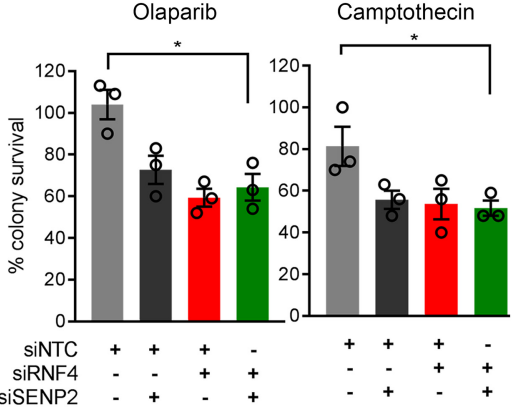

L

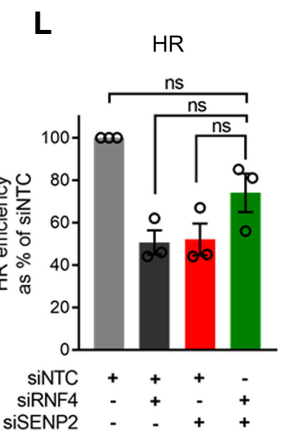

M
N

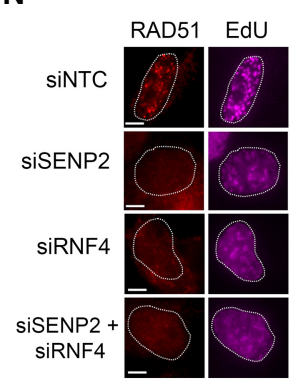

Figure 4. SENP2 is not relevant to S-phase clearance of MDC1. $(A, B)$ HeLa treated with siNTC (black) or siSENP2 (blue) for 72 h, pulsed with $10 \mu \mathrm{M}$ EdU $1 \mathrm{~h}$ prior to IR (4 Gy). Cells were fixed at indicated times and subjected to Click-It labeling with $647 \mathrm{~nm}$ azide to detect EdU incorporation into nascent chromatin. MDC1 foci per cell in EdU-negative $(A)$ and EdU-positive $(S$ phase) $(B)$ cells. Fifty cells were scored per condition from a total of three experiments. Error bars represent SEM. $(C)$ Representative images relating to $A$ and $B$. $(D)$ Western blot showing MDC1 knockdown and expression in HeLa ${ }^{\text {FlpIn }}$ myc-MDC1 wild type or K1840R (KR). (E-G) HeLa ${ }^{\text {FlpIn }}$ myc-MDC1 ${ }^{\text {WT }}$ (blue) and K1840R (green) cells siRNA depleted for endogenous MDC1 and treated with doxycycline to induce MDC1. After $72 \mathrm{~h}$, cells were treated with 2 Gy of IR $(E), 10 \mu \mathrm{M}$ olaparib $(F)$, or $2.5 \mu \mathrm{M} \mathrm{CPT}(G)$ for $2 \mathrm{~h}$ and subjected to colony survival analysis. $n=3$. $(H) \mathrm{HeLa}$ myc-MDC1 ${ }^{\mathrm{WT}}$ (blue) and K1840R (green) cells siRNA depleted for endogenous MDC1 and treated with doxycycline to induce mycMDC1. After $72 \mathrm{~h}$, cells were pulsed with $10 \mu \mathrm{M} \mathrm{EdU} 1 \mathrm{~h}$ prior to $4 \mathrm{~Gy}$ of IR. Cells were fixed $2 \mathrm{~h}$ after IR and subjected to Click-It labeling with 647-nm azide to detect EdU incorporation into nascent chromatin and stained with RAD51. $n=100$ EdU ${ }^{+}$cells. $(I)$ HeLa treated with doxycycline to induce expression of myc-MDC1 ${ }^{\mathrm{WT}}$ or K1840R for $72 \mathrm{~h}$. One hour prior to $4 \mathrm{~Gy}$ of IR, cells were pulsed with EdU and fixed $2 \mathrm{~h}$ later. Cells (100 from a total of three experiments) were scored for 53BP1 foci per cell in EdU $\mathrm{U}^{-/+}$cells. $(J, K)$. Colony survival in HeLa treated with indicated siRNA and $10 \mu \mathrm{M}$ olaparib for $2 \mathrm{~h}(J)$ or $2.5 \mu \mathrm{M}$ CPT for $2 \mathrm{~h}(K) . n=3$. $(L)$ U2OS-DR3 homologous recombination reporter cells treated with siRNA for $24 \mathrm{~h}$ prior to transfection with I-Sce-I nuclease and RFP (to control for transfection efficiency) for a further $48 \mathrm{~h}$. The percentage of RFP/GFP-positive cells relative to siNTC is shown for three experiments. $(M)$ HeLa depleted for indicated siRNA for $72 \mathrm{~h}$ were pulsed with $10 \mu \mathrm{M}$ EdU for $1 \mathrm{~h}$ prior to $4 \mathrm{~Gy}$ of irradiation. Cells were fixed $4 \mathrm{~h}$ later and RAD51 foci counted in EdUpositive cells. $n=100$. (N) Images relating to $M$. 


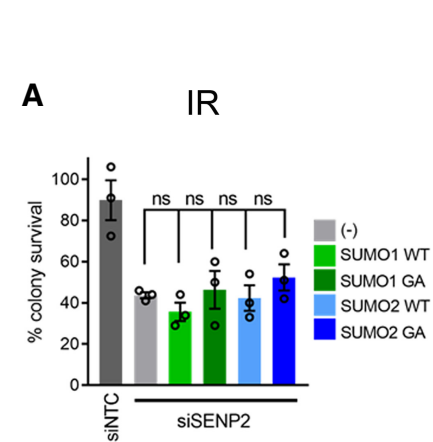

B

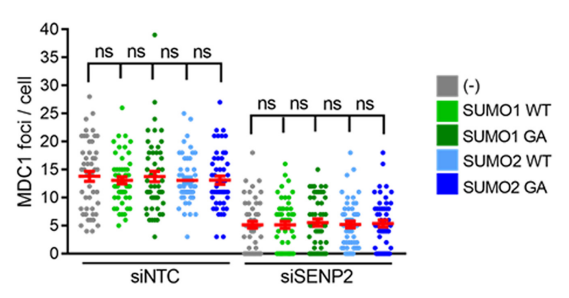

D

Camptothecin

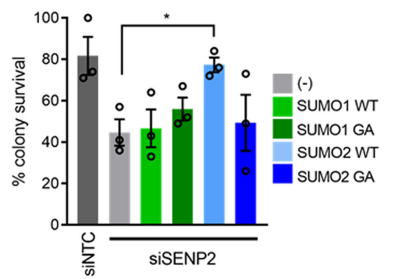

E

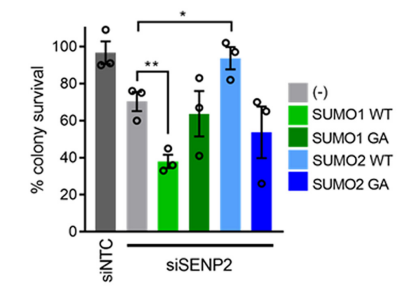

$\mathbf{F}$
C

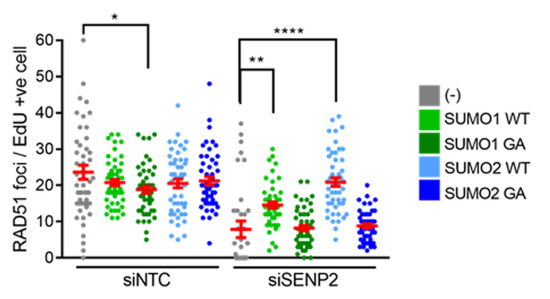

\section{G}

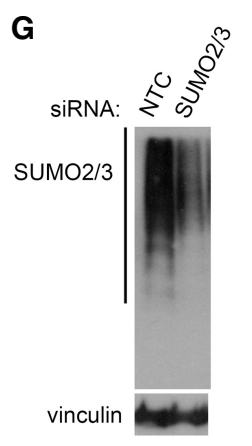

H

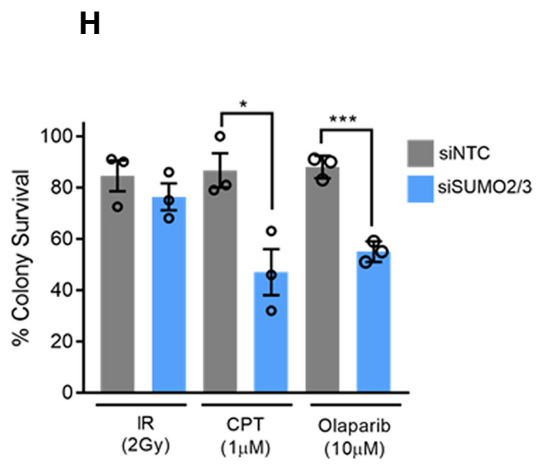

$$
\text { I }
$$

I

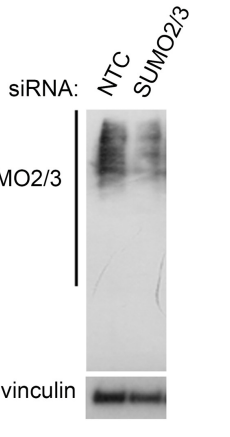

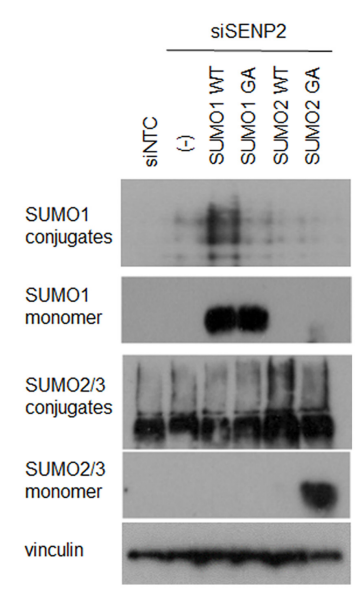

J

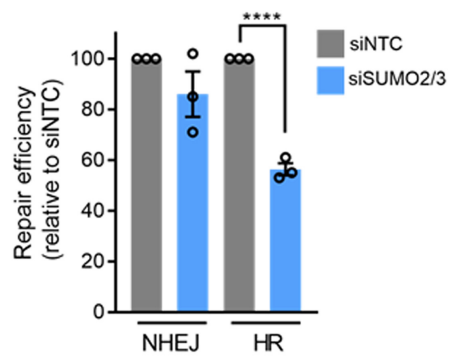

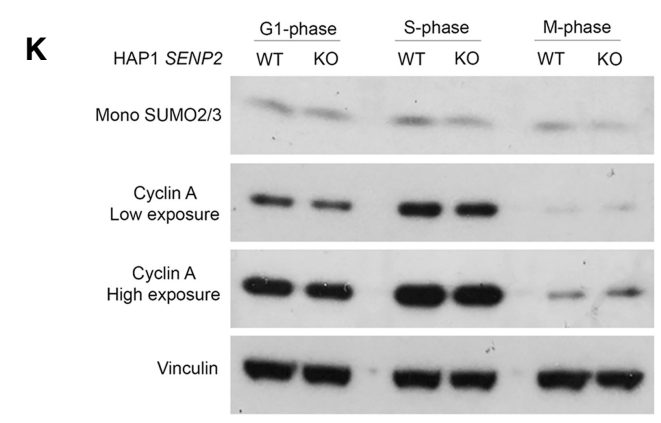

Figure 5. HR is sensitive to the supply of SUMO. $(A)$ Colony survival after 2 Gy IR in HeLa ${ }^{\text {FlpIn }}$ 6xHis-myc SUMO in siNTC/siSENP2depleted cells. GA indicates diglycine $\rightarrow$ alanine mutants in SUMO isoforms that prevent conjugation. $n=3$. $(B)$ HeLa treated with siRNA for $24 \mathrm{~h}$ before transfection with myc-SUMO; $48 \mathrm{~h}$ later cells were treated with $4 \mathrm{~Gy}$, fixed at $4 \mathrm{~h}$, immunostained, and scored for MDC1 in myc-SUMO expressing cells. $n=100$. $(C)$ Western blot of SUMO conjugates relating to $(A, B)$. $(D, E)$ As for $A$ but using $1 \mu M$ CPT $(D)$ or $10 \mu \mathrm{M}$ olaparib $(E)$ for $2 \mathrm{~h}$ before plating for colony survival. $n=3$. $(F)$. HeLa treated with siRNA for $24 \mathrm{~h}$ before transfection with mycSUMO for $48 \mathrm{~h}$. Cells were incubated with $10 \mu \mathrm{M}$ EdU $30 \mathrm{~min}$ prior to $4 \mathrm{~Gy}$ of IR, fixed at $4 \mathrm{~h}$, and immunostained for RAD51 and myc to detect myc-SUMO-expressing cells. $n=100$. Only EdU ${ }^{+}$myc-SUMO-positive cells were counted for RAD51 focus number. $(G)$ Western blot showing partial depletion of SUMO2/3 conjugates in HeLa. $(H)$ Colony survival in HeLa depleted with siNTC or siSUMO2/3 followed by treatment with 2 Gy of IR, $1 \mu \mathrm{M}$ CPT, or $10 \mu \mathrm{M}$ olaparib. $(I)$. Western blot showing SUMO2/3 knockdown. (J) U2OS HR and NHEJ reporters treated with siNTC or siSUMO2/3 and transfected with $i$-Sce-I and RFP for 72 h. HR and NHEJ efficiency was set at $100 \%$ for siNTC. (K) HAP1 cells synchronized into G1-, S-, and M-enriched populations; lysed; and probed for free SUMO2/3. Cyclin A1 is a late S-phase marker. 
A

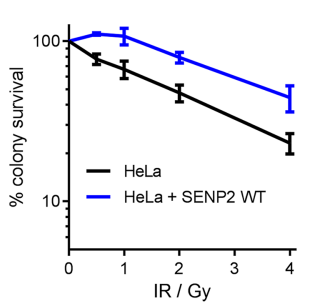

E

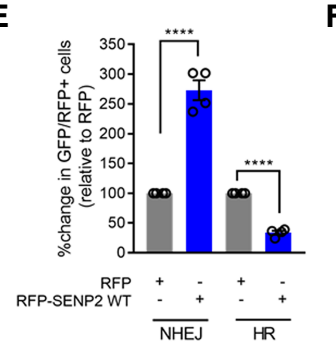

H
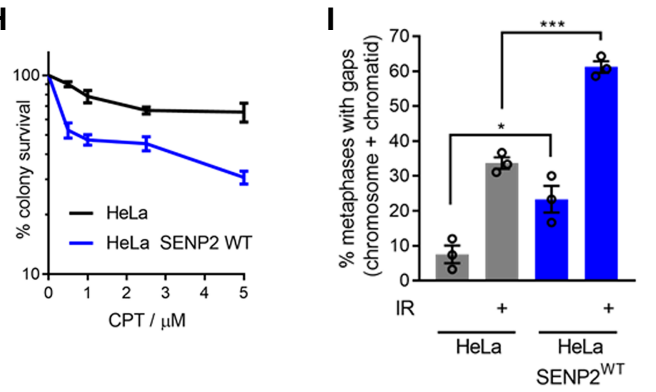

C

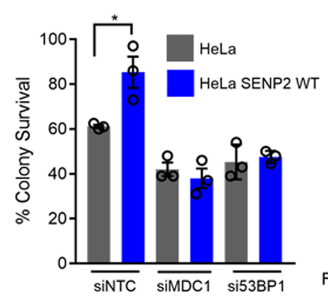

$\mathbf{F}$

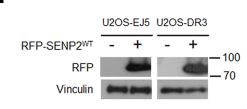

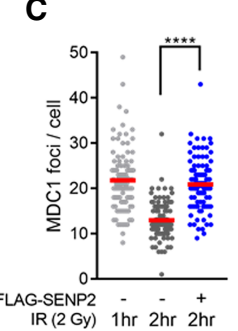

G

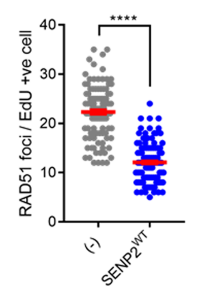

D FLAG

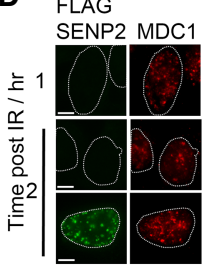

Figure 6. SENP2 overexpression disrupts responses to DSBs. $(A, B) \mathrm{HeLa}^{\mathrm{FlpIn}}$ SENP2 ${ }^{\mathrm{WT}}$ mock or doxycycline (to induce SENP2 expression) for $72 \mathrm{~h}$ were treated with indicated dose of IR and subjected to colony survival analysis. (B) Colony assay performed as for $A$ but with siRNA transfection concurrent with doxycycline addition. IR $=2$ Gy. $n=3$. $(C, D)$ HeLa transfected with SENP2 ${ }^{\mathrm{WT}}$ for 48 $\mathrm{h}$ prior to 4 Gy IR and fixation $2 \mathrm{~h}$ later. $\mathrm{MDC1}$ foci per cell were scored in FlagSENP2-positive cells. $n=100 .(D)$ Representative images of $C .(E, F) \mathrm{HR}$ and NHEJ U2OS reporters expressing RFP or RFP-SENP2 and I-Sce1 GFP-positive cells were normalized to RFP-transfection efficiency. Percentage repair is given compared with NTC. $(F)$ Western blot showing expression of RFP-SENP2. $n=4$. $(G)$ HeLa transfected with SENP2 ${ }^{\mathrm{WT}}$ for $48 \mathrm{~h}$ prior to 30 min of EdU pulse and 4 Gy of IR followed by fixation $4 \mathrm{~h}$ later. One-hundred EdU/ Flag-SENP2-positive and 100 EdU/untransfected cells were scored for RAD51 foci across three experiments. $(H)$ As for $A$ but using $2 \mathrm{~h}$ of treatment of $2.5 \mu \mathrm{M} \mathrm{CPT}$ for $2 \mathrm{~h}$ prior to plating. (I) HeLa SENP2 ${ }^{\mathrm{WT}}$ or HeLa treated with doxycycline for $72 \mathrm{~h}$ prior to IR $2 \mathrm{~Gy}$. Eighteen hours later, cells were treated with colcimid for $6 \mathrm{~h}$ and processed for metaphase spread analysis. Data show percentage metaphases with chromosome/chromatid gaps from three experiments. Error bars represent SEM.

integrated substrate (Fig. 6E,F). Thus, increased SENP2 results in slower MDC1 clearance correlating with increased IR resistance and improved NHEJ. Increased expression of SENP2 reduces high-molecular-weight SUMO conjugates (Supplemental Fig. S4E, F), leading us to speculate whether persistent removal of SUMO may also influence HR. High SENP2 expression resulted in reduced HR reporter product, reduced RAD51 foci, and reduced CPT resistance (Fig. 6G,H). Examination of chromosome aberrations in cells acutely overexpressing SENP2 showed increased chromosomal gaps following IR, suggesting an overall reduced repair ability despite improved IR resistance (Fig. 6I).

\section{Discussion}

The sequential action of PTMs is essential for the proper cellular response to DSBs. While cross-talk between SUMOylation and ubiquitination is important for the integration of signaling cues for the response, the extent of a role for deSUMOylation was poorly defined. Here we provide evidence that deSUMOylation is required prior to the onset of DSB signaling to govern correct PTM timing following damage. Mechanistically, we identified two distinct pathways in which deSUMOylation is required (Supplemental Fig. S7A,B).

We show that interaction between MDC1 and SENP2 in untreated cells is associated with MDC1-hypoSUMOylation and with Ub signaling in the damage response. In the absence of SENP2, PIAS4-mediated SUMOylation facilitates rapid RNF4-VCP-mediated MDC1 turnover and a failure of downstream signaling. MDC1 SUMOylation and RNF4-processing is induced on chromatin (Luo et al. 2012), so one question arising from our study is why constitutive interaction with a SUMO protease is needed? MDC1-SUMO is detected in untreated cells /Galanty et al. 2012; Luo et al. 2012; Yin et al. 2012; Vyas et al. 2013; Hendriks and Vertegaal 2015; Hendriks et al. 2015 ), suggesting a constitutive propensity to modification even in the presence of SENP2. Thus, interaction with a SUMO protease may be needed to prevent the accumulation of a heavily modified protein at DSBs capable of driving its own removal.

We show that DDR signaling via ATM and conserved coiled-coil region (amino acids 203-228) in SENP2 is needed to release MDC1-SENP2 interactions following IR and to allow subsequent MDC1 SUMOylation needed for its eventual clearance from damaged chromatin. The SENP family of SUMO proteases contain relatively few functionally annotated domains outside of the C-terminal catalytic regions (Mukhopadhyay and Dasso 2007). How the coiled-coil allows IR-regulated dissociation remains to be discovered. Its conservation in evolution as far as chicken and zebrafish (Supplemental Fig. S4B) suggests an important role for the motif.

We found that the role of SENP2 in HR repair has no relationship with the MDC1-processing pathway, that MDC1 foci are cleared in S-phase cells at the same 
kinetics with or without SENP2, and that MDC1 in which the main SUMO acceptor site is mutated is also cleared in S-phase cells at the same rate as wild-type protein. MDC1depleted cells complemented with the K1840R mutant were IR-sensitive, as described previously (Luo et al. 2012); however, they exhibit normal levels of RAD51 foci in S-phase cells and exhibit both olaparib and CPT resistance, suggesting adequate HR repair. A previous report correlated RNF4 activation with altered MDC1 kinetics in S phase (Luo et al. 2015). We suggest that the altered MDC1 kinetics in S-phase RNF4 depleted or mutant cells may be due to indirect regulation by RNF4 through other aspects of DNA repair. However, the previous finding that MDC1-K1840R significantly impacts HR repair (Luo et al. 2012) was not supported by the current study. We cannot rule out that another SUMO site is significant, although, if such a site were SUMO-modified for RNF4 interaction, it would differ in its response to SENP2.

Instead, measures of HR repressed in SENP2-deficient cells are rescued by the expression of exogenous SUMO2/3. SUMO conjugation is required for both main pathways of DSB repair so that a total loss of SUMO availability/conjugation restricts both mechanisms (Galanty et al. 2009; Morris et al. 2009). What is striking in our findings is evidence for a level of SUMO availability at which NHEJ can function but HR cannot. The degree to which each repair process captures available SUMO is not known. The differential requirement may reflect the greater number of SUMOylated factors in HR over NHEJ, a greater need for group modification in $\mathrm{HR}$, or a greater need for the promotion of particular interactions, for example, between BLM, RPA, and RAD51 (Eladad et al. 2005; Ouyang et al. 2009; Dou et al. 2010; Galanty et al. 2012; Bologna et al. 2015; Hendriks and Vertegaal 2016; Garvin and Morris 2017). Alternatively, cells in S phase may have a greater need for available SUMO in replicative processes, reducing availability for $\mathrm{HR}$, which is somewhat supported by our data (Fig. 5K).

In the HeLa ${ }^{\text {FlpIn }}$ cells used in the current study we detected no free SUMO pool and no accumulation of exogenous SUMO2 in a free SUMO2/3 pool, suggesting that the increase in SUMO2 availability was immediately captured within conjugates. In some cell types, the vast majority of SUMO exists in conjugates; for example, shifting from $93 \%$ of SUMO2/3 in conjugates to $96 \%$ and $98 \%$ on MG132 and heat shock, respectively, in HEK293T cells (Hendriks et al. 2018). In these contexts, induced SUMO conjugation in stress responses may be reliant on SUMO synthesis and recycling from SUMOylated proteins.

We also show that acute, high-level expression of SENP2 results in increased NHEJ correlating with extended MDC1 focus longevity and 53BP1-dependent IR resistance, consistent with extended defense of MDC1SUMO. SENP2 overexpression also reduces global SUMO conjugates, and we speculate strips SUMO from HR proteins during the damage response. In both pathways SENP2 levels dramatically influence repair outcomes.

Many cancers have altered SUMOylation (Seeler and Dejean 2017) and SENP2 transcription can be up-regulat- ed by NF-kb, which is commonly activated during cancer development (Lee et al. 2011). SENP2 is one of several genes on the amplified region of chromosome $3 \mathrm{q}$ with the capacity to influence survival to DNA-damaging therapeutics. Evidence of SUMO-pathway addiction has been found in Myc- and Ras-driven cancers (Kessler et al. 2012; Yu et al. 2015), while those with low SUMOylation may be sensitive to further targeting of the SUMO system (Licciardello et al. 2015). Differential needs for SUMO conjugation and deconjugation therefore could expose tumorspecific vulnerabilities. SUMO E1 and E2 inhibitors have been described (Lu et al. 2010; Kumar et al. 2016; He et al. 2017), and our data imply that partial SUMO conjugation inhibition could disable HR but not NHEJ, increasing sensitivity to HR-directed therapies. Common $3 q$-amplified tumors such as those in the aerodigestive track cancers often receive postoperative radiotherapy, so further investigation into targeting altered DNA repair that may occur as a result of $3 \mathrm{q}$ amplification is warranted.

In summary, the need for the SUMO protease, SENP2, in aspects of mammalian DSB repair presented here reveal unexpected requirements for SUMO deconjugation and its regulation in the DNA damage response. They place the need for the activity largely in undamaged cells before the stress of DSBs occurs. We found deSUMOylation by SENP2 first prevents engagement of RNF4-VCP with MDC1, restricting an "over before it has begun" repair response and secondly promotes SUMO supply, critical to the completion of HR. Increased SENP2 expression therefore dramatically dysregulates DSB repair mechanisms.

\section{Materials and Methods}

Colony survival assays

Cells were plated at $2.5 \times 10^{5}$ cells per milliliter in a 24 -well plate. For siRNA transfections, cells were transfected $24 \mathrm{~h}$ after plating for an additional $48 \mathrm{~h}$. For overexpression, cells were treated with $1 \mu \mathrm{g} / \mathrm{mL}$ doxycycline for $72 \mathrm{~h}$. Cells were treated with the indicated dose of DNA-damaging agent prior to plating at limiting dilution in six-well plates to form colonies and grown for $10 \mathrm{~d}$ (three wells per technical repeat). Colonies were stained with $0.5 \%$ crystal violet (BDH Chemicals) in 50\% methanol and counted. Colony survival was calculated as the percentage change in colonies versus untreated matched controls. Graphs shown are combined data from a minimum of three independent experiments and error bars show SEM.

\section{Transfections}

siRNA transfections (10 $\mathrm{nM}$ ) were performed using Dharmafectl (Dharmacon) and DNA plasmids using FuGENE $6(3 \mu \mathrm{L}$ of FuGENE:1 $\mu$ g of DNA) (Promega) following the manufacturer's protocols. SMARTPools were from Dharmacon and individual sequences were from Sigma. See Supplemental Table 3 for siRNA sequences. Cells were grown for 48-72 h after transfection before treatment and harvesting.

Drug treatments

Irradiation was performed with a Gamma-cell 1000 Elite $\left(\mathrm{Cs}^{137}\right)$ radiation source. The following chemicals were used: $0.1 \mu \mathrm{M}$ 
CB-5083/VCPi, $10 \mu \mathrm{M}$ olaparib (Selleck Chemicals), $1 \mu \mathrm{M} \mathrm{CPT,}$ $10 \mu \mathrm{M}$ MG132, $0.05 \mu \mathrm{g} / \mathrm{mL}$ colcimid, $1 \mu \mathrm{g} / \mathrm{mL}$ aphidocolin, 2 $\mathrm{mM}$ thymidine, $150 \mathrm{ng} / \mathrm{mL}$ nocodazole (Sigma), $10 \mu \mathrm{M}$ EdU (Life Technologies), and KU55933 (Abcam, ab120637).

\section{NHEJ and HR assays}

U2OS-DR3-GFP (gene conversion), and U2OS-EJ5-GFP (NHEJ) were a generous gift from Jeremy Stark (City of Hope, Duarte, USA). U20S reporter cell lines were simultaneously cotransfected with siRNA using Dharmafect1 (Dharmacon) and DNA (RFP, or Flag-SENP2 and I-Sce1 endonuclease expression constructs) using FuGene6 (Promega), respectively. After $16 \mathrm{~h}$, the media was replaced and cells were grown for a further $48 \mathrm{~h}$ before fixation in $2 \%$ PFA. RFP and GFP double-positive cells were scored by FACS analysis using a CyAn flow cytometer and a minimum of 10,000 cells counted. Data was analyzed using Summit 4.3 software. Each individual experiment contained three technical repeats and normalized to siRNA controls or wild-typecomplemented cells. Graphs shown are combined data from a minimum of three independent experiments and error bars show standard error.

\section{Immunofluorescence}

Cells were plated on 13-mm circular glass coverslips at a density of $5 \times 10^{4}$ cells per milliliter and treated as required. For RPA and RAD51, cells were labeled with $10 \mu \mathrm{M}$ EdU $1 \mathrm{~h}$ prior addition of DNA damage. For staining, cells were pre-extracted in CSK buffer (100 mM sodium chloride, $300 \mathrm{mM}$ sucrose, $3 \mathrm{mM} \mathrm{MgCl} 2,10$ $\mathrm{mM}$ PIPES at $\mathrm{pH} 6.8$ ) for $1 \mathrm{~min}$ at room temperature, For all other stainings, cells were first fixed in 4\% PFA and permeabilized with $0.5 \%$ Triton X-100 in PBS. After blocking in 10\% FCS, cells were incubated with primary antibody for $1 \mathrm{~h}$ (unless stated otherwise) and with secondary Alexa fluor antibodies for $1 \mathrm{~h}$. EdU was visualized by Click-iT chemistry according to the manufacturer's protocols (Life Technologies) with Alexa-647-azide. The DNA was stained using Hoechst at 1:20,000. In some images, the DNA stain was drawn around (but not shown) to illustrate the location of the nucleus.

With the exception of Figure 1, G and $\mathrm{H}$, all immunofluorescent staining was imaged using the Leica DM6000B microscope using a HBO lamp with a 100-W mercury short-arc UV bulb light source and four filter cubes (A4, L5, N3, and Y5) to produce excitations at wavelengths $360,488,555$, and $647 \mathrm{~nm}$, respectively. Images were captured at each wavelength sequentially using the plan apochromat HCX $100 \times / 1.4$ oil objective at a resolution of $1392 \times 1040$ pixels. Detection of SUMO IRIF was performed according to Morris et al. (2009).

\section{Cell lines}

The growth conditions and vendors for all cell lines are detailed in Supplemental Table 2. FlpIn stable cell lines were generated using HEK293 ${ }^{\text {TrEx-FlpIn }}$ (Invitrogen) and $\mathrm{HeLa}^{\text {FlpIn }}$ (a gift from Grant Stewart, University of Birmingham) cells transfected with pcDNA5/FRT/TO-based vectors and the recombinase pOG44 (Invitrogen) using FuGene6 (Promega). After 48 h, cells were placed into $100 \mu \mathrm{g} / \mathrm{mL}$ hygromycin selection medium and grown until colonies formed on plasmid transfected plates but not controls. HAP1 SENP2 knockout cells (128-base-pair deletion in exon 3, HZGHC002974c003) and parental cells were from Horizon Discovery and were cultured according to manufacturer's instructions.

\section{Coimmunoprecipitation}

HEK $293^{\text {FlpIn }}$ myc-MDC1 ${ }^{\text {WT }}$ were seeded on $10-\mathrm{cm}$ plates in the presence of $1 \mu \mathrm{g} / \mathrm{mL}$ doxycycline for $24 \mathrm{~h}$ prior to transfection with $3 \mu \mathrm{g}$ of Flag-SENP2 per plate for a further $48 \mathrm{~h}$. Cells were treated with $4 \mathrm{~Gy}$ of IR and pelleted $1 \mathrm{~h}$ later in cold PBS. Cell pellets were lysed in $0.5 \mathrm{~mL}$ hypotonic buffer $(10 \mathrm{mM}$ HEPES at $\mathrm{pH}$ $7.8,10 \mathrm{mM} \mathrm{KCl}, 1.5 \mathrm{mM} \mathrm{MgCl}_{2}, 340 \mathrm{mM}$ sucrose, $10 \%$ glycerol $0.2 \%$ NP40, protease, phosphatase inhibitor cocktails) for $5 \mathrm{~min}$ on ice and centrifuged at $3000 \mathrm{rpm}$ for $3 \mathrm{~min}$. The nuclear pellet was lysed in nuclear buffer $(0.05 \%$ NP40, $50 \mathrm{mM}$ Tris $\mathrm{pH} 8,300$ $\mathrm{mM} \mathrm{NaCl}$, protease and phosphatase inhibitor cocktails) and rotated for $30 \mathrm{~min}$ at $4^{\circ} \mathrm{C}$. Lysates were briefly sonicated and clarified at 12,000 rpm for $10 \mathrm{~min}$ to remove debris. Cleared lysates $(0.9 \mathrm{~mL})$ were incubated with either myc (Thermo Fisher) or M2 (Sigma) agarose $\left(20-\mu \mathrm{L}\right.$ packed bead volume) for $16 \mathrm{~h}$ at $4^{\circ} \mathrm{C}$ with rotation. Beads were washed three times with NETN buffer (100 mM NaCl, $20 \mathrm{mM}$ Tris- $\mathrm{HCl}$ at $\mathrm{pH} 8,0.5 \mathrm{mM}$ EDTA, 0.5\% NP40) before elution with $4 \times$ Lamelli buffer.

Supplemental methods

Methods describing cloning, in vitro assays, pull-downs, metaphase spreads, and cell cycle synchronization are in the Supplemental Material. Supplemental Tables 1-4 contain details on antibodies, cell lines, siRNA, and oligonucleotide sequences, respectively.

\section{Statistics}

Unless stated otherwise, all statistical analysis was by two-sided Student's $t$-test throughout $\left(P<0.05\left[{ }^{*}\right], P<0.01\left[{ }^{* *}\right], P<0.005\right.$ $\left[{ }^{* * *}\right]$, and $\left.P<0.001\left[{ }^{* * *}\right]\right)$. All center values are given as the mean, and all error bars are SEM. Data were analyzed using Graphpad Prism 7.03.

\section{Quantification}

All Western blot or image analysis for quantification was done using ImageJ unless specified otherwise.

\section{Acknowledgments}

We thank Jeremy Stark for the U2OS reporter cell lines (City of Hope, USA), and the Microscopy, Imaging, and FACS services at Birmingham University in the Tech Hub facility for equipment support and maintenance. Grant funding for this project was as follows. Wellcome Trust grant 206343/Z/17/Z to A.J.G., A.K.W., A.S.C., and M.J.; Cancer Research UK (CRUK) grant C8820/A19062 to A.J.G., H.R.S., R.M.D.; Breast Cancer Now grant 2015MayPR499 to K.S.; and CRUK Centre training grant to M.D.-M.

Author contributions: Cloning and generation of stable cell lines was performed by A.J.G., A.K.W., and R.M.D. Coimmunoprecipitation and pull-downs were performed by A.J.G., A.K.W., and A.S.C. Immunofluorescence analysis was performed by A.J.G., A.K.W., M.D.-M., and H.R.S. FACS analysis was performed by A.K.W. and R.M.D. Metaphase spreads were performed by K.S. and J.F.B. Immunoblots were performed by A.J.G., A.K.W., A.S.C., H.L.M., and G.E.R. The manuscript was written and the project was conceived by A.J.G. and J.R.M. All authors reviewed the manuscript. 


\section{References}

Bologna S, Altmannova V, Valtorta E, Koenig C, Liberali P, Gentili C, Anrather D, Ammerer G, Pelkmans L, Krejci L, et al. 2015. Sumoylation regulates EXO1 stability and processing of DNA damage. Cell Cycle 14: 2439-2450. doi:10.1080/ 15384101.2015.1060381

Butler LR, Densham RM, Jia J, Garvin AJ, Stone HR, Shah V, Weekes D, Festy F, Beesley J, Morris JR. 2012. The proteasomal de-ubiquitinating enzyme $\mathrm{POH} 1$ promotes the doublestrand DNA break response. EMBO J 31: 3918-3934. doi:10 $.1038 /$ emboj.2012.232

Cancer Genome Atlas Network. 2015. Comprehensive genomic characterization of head and neck squamous cell carcinomas. Nature 517: 576-582. doi:10.1038/nature14129

Chapman JR, Jackson SP. 2008. Phospho-dependent interactions between NBS1 and MDC1 mediate chromatin retention of the MRN complex at sites of DNA damage. EMBO Rep 9: 795-801. doi:10.1038/embor.2008.103

Chen YJ, Chuang YC, Chuang CN, Cheng YH, Chang CR, Leng $\mathrm{CH}$, Wang TF. 2016. S. cerevisiae Mre11 recruits conjugated SUMO moieties to facilitate the assembly and function of the Mre11-Rad50-Xrs2 complex. Nucleic Acids Res 44: 2199-2213. doi:10.1093/nar/gkv1523

Chow KH, Elgort S, Dasso M, Powers MA, Ullman KS. 2014. The SUMO proteases SENP1 and SENP2 play a critical role in nucleoporin homeostasis and nuclear pore complex function. Mol Biol Cell 25: 160-168. doi:10.1091/mbc.e13-05-0256

Chroma K, Mistrik M, Moudry P, Gursky J, Liptay M, Strauss R, Skrott Z, Vrtel R, Bartkova J, Kramara J, et al. 2017. Tumors overexpressing RNF168 show altered DNA repair and responses to genotoxic treatments, genomic instability and resistance to proteotoxic stress. Oncogene 36: 2405-2422. doi:10.1038/onc.2016.392

Danielsen JR, Povlsen LK, Villumsen BH, Streicher W, Nilsson J, Wikstrom M, Bekker-Jensen S, Mailand N. 2012. DNA damage-inducible SUMOylation of HERC2 promotes RNF8 binding via a novel SUMO-binding Zinc finger. J Cell Biol 197: 179-187. doi:10.1083/jcb.201106152

Dantuma NP, Acs K, Luijsterburg MS. 2014. Should I stay or should I go: VCP/p97-mediated chromatin extraction in the DNA damage response. Exp Cell Res 329: 9-17. doi:10.1016/ j.yexcr.2014.08.025

Doil C, Mailand N, Bekker-Jensen S, Menard P, Larsen DH, Pepperkok R, Ellenberg J, Panier S, Durocher D, Bartek J, et al. 2009. RNF168 binds and amplifies ubiquitin conjugates on damaged chromosomes to allow accumulation of repair proteins. Cell 136: 435-446. doi:10.1016/j.cell.2008.12.041

Dou H, Huang C, Singh M, Carpenter PB, Yeh ETH. 2010. Regulation of DNA repair through DeSUMOylation and SUMOylation of replication protein A complex. Mol Cell 39: 333345. doi:10.1016/j.molcel.2010.07.021

Eladad S, Ye TZ, Hu P, Leversha M, Beresten S, Matunis MJ, Ellis NA. 2005. Intra-nuclear trafficking of the BLM helicase to DNA damage-induced foci is regulated by SUMO modification. Hum Mol Genet 14: 1351-1365. doi:10.1093/hmg/ ddi 145

Galanty Y, Belotserkovskaya R, Coates J, Polo S, Miller KM, Jackson SP. 2009. Mammalian SUMO E3-ligases PIAS1 and PIAS4 promote responses to DNA double-strand breaks. Nature 462: 935-939. doi:10.1038/nature08657

Galanty Y, Belotserkovskaya R, Coates J, Jackson SP. 2012. RNF4, a SUMO-targeted ubiquitin E3 ligase, promotes DNA doublestrand break repair. Genes Dev 26: 1179-1195. doi:10.1101/ gad.188284.112
Garvin AJ, Morris JR. 2017. SUMO, a small, but powerful, regulator of double-strand break repair. Philos Trans $R$ Soc Lond B Biol Sci 372: 20160281. doi:10.1098/rstb.2016.0281

Garvin AJ, Densham R, Blair-Reid SA, Pratt KM, Stone HR, Weekes D, Lawrence KJ, Morris JR. 2013. The deSUMOylase SENP7 promotes chromatin relaxation for homologous recombination DNA repair. EMBO Rep 14: 975-983. doi:10 .1038/embor.2013.141

Goeres J, Chan PK, Mukhopadhyay D, Zhang H, Raught B, Matunis MJ. 2011. The SUMO-specific isopeptidase SENP2 associates dynamically with nuclear pore complexes through interactions with karyopherins and the Nup107-160 nucleoporin subcomplex. Mol Biol Cell 22: 4868-4882. doi:10 $.1091 / \mathrm{mbc} . \mathrm{e} 10-12-0953$

Hang J, Dasso M. 2002. Association of the human SUMO-1 protease SENP2 with the nuclear pore. J Biol Chem 277: 1996119966. doi:10.1074/jbc.M201799200

Hang LE, Lopez CR, Liu X, Williams JM, Chung I, Wei L, Bertuch AA, Zhao X. 2014. Regulation of Ku-DNA association by Yku70 C-terminal tail and SUMO modification. I Biol Chem 289: 10308-10317. doi:10.1074/jbc.M113.526178

He X, Riceberg J, Soucy T, Koenig E, Minissale J, Gallery M, Bernard H, Yang X, Liao H, Rabino C, et al. 2017. Probing the roles of SUMOylation in cancer cell biology by using a selective SAE inhibitor. Nat Chem Biol 13: 1164-1171. doi:10.1038/ nchembio. 2463

Hecker CM, Rabiller M, Haglund K, Bayer P, Dikic I. 2006. Specification of SUMO1- and SUMO2-interacting motifs. I Biol Chem 281: 16117-16127. doi:10.1074/jbc.M512757200

Hendriks IA, Vertegaal AC. 2015. SUMO in the DNA damage response. Oncotarget 6: 15734-15735. doi:10.18632/oncotarget .4605

Hendriks IA, Vertegaal AC. 2016. A comprehensive compilation of SUMO proteomics. Nat Rev Mol Cell Biol 17: 581-595. doi:10.1038/nrm.2016.81

Hendriks IA, Treffers LW, Verlaan-de Vries M, Olsen JV, Vertegaal AC. 2015. SUMO-2 orchestrates chromatin modifiers in response to DNA damage. Cell Rep doi:10.1016/j.celrep .2015.02.033.

Hendriks IA, Lyon D, Su D, Skotte NH, Daniel JA, Jensen LJ, Nielsen ML. 2018. Site-specific characterization of endogenous SUMOylation across species and organs. Nat Commun 9: 2456. doi:10.1038/s41467-018-04957-4

Ismail IH, Gagné JP, Caron MC, McDonald D, Xu Z, Masson JY, Poirier GG, Hendzel MJ. 2012. CBX4-mediated SUMO modification regulates BMI1 recruitment at sites of DNA damage. Nucleic Acids Res 40: 5497-5510. doi:10.1093/nar/gks222

Jentsch S, Psakhye I. 2013. Control of nuclear activities by substrate-selective and protein-group SUMOylation. Annu Rev Genet 47: 167-186. doi:10.1146/annurev-genet-111212133453

Kessler JD, Kahle KT, Sun T, Meerbrey KL, Schlabach MR, Schmitt EM, Skinner SO, Xu Q, Li MZ, Hartman ZC, et al. 2012. A SUMOylation-dependent transcriptional subprogram is required for Myc-driven tumorigenesis. Science 335: 348 353. doi:10.1126/science.1212728

Kumar R, Cheok CF. 2017. Dynamics of RIF1 SUMOylation is regulated by PIAS4 in the maintenance of Genomic Stability. Sci Rep 7: 17367. doi:10.1038/s41598-017-16934-w

Kumar A, Ito A, Hirohama M, Yoshida M, Zhang KY. 2016. Identification of new SUMO activating enzyme 1 inhibitors using virtual screening and scaffold hopping. Bioorg Med Chem Lett 26: 1218-1223. doi:10.1016/j.bmcl.2016.01.030

Kumar R, González-Prieto R, Xiao Z, Verlaan-de Vries M, Vertegaal ACO. 2017. The STUbL RNF4 regulates protein group 
SUMOylation by targeting the SUMO conjugation machinery. Nat Commun 8: 1809. doi:10.1038/s41467-017-01900-x

Kung CC, Naik MT, Wang SH, Shih HM, Chang CC, Lin LY, Chen CL, Ma C, Chang CF, Huang TH. 2014. Structural analysis of poly-SUMO chain recognition by the RNF4-SIMs domain. Biochem J 462: 53-65. doi:10.1042/BJ20140521

Lamoliatte F, Caron D, Durette C, Mahrouche L, Maroui MA, Caron-Lizotte O, Bonneil E, Chelbi-Alix MK, Thibault P. 2014. Large-scale analysis of lysine SUMOylation by SUMO remnant immunoaffinity profiling. Nat Commun 5: 5409. doi:10.1038/ncomms6409

Lee MH, Mabb AM, Gill GB, Yeh ET, Miyamoto S. 2011. NF-кB induction of the SUMO protease SENP2: a negative feedback loop to attenuate cell survival response to genotoxic stress. Mol Cell 43: 180-191. doi:10.1016/j.molcel.2011.06.017

Li YJ, Stark JM, Chen DI, Ann DK, Chen Y. 2010. Role of SUMO: SIM-mediated protein-protein interaction in non-homologous end joining. Oncogene 29: 3509-3518. doi:10.1038/onc .2010 .108

Li Y, Luo K, Yin Y, Wu C, Deng M, Li L, Chen Y, Nowsheen S, Lou Z, Yuan J. 2017. USP13 regulates the RAP80-BRCA1 complex dependent DNA damage response. Nat Commun 8: 15752. doi:10.1038/ncomms 15752

Licciardello MP, Mullner MK, Dürnberger G, Kerzendorfer C, Boidol B, Trefzer C, Sdelci S, Berg T, Penz T, Schuster M, et al. 2015. NOTCH1 activation in breast cancer confers sensitivity to inhibition of SUMOylation. Oncogene 34: 37803790. doi:10.1038/onc.2014.319

Lok GT, Sy SM, Dong SS, Ching YP, Tsao SW, Thomson TM, Huen MS. 2011. Differential regulation of RNF8-mediated Lys48- and Lys63-based poly-ubiquitylation. Nucleic Acids Res 40: 196-205. doi:10.1093/nar/gkr655

Lu X, Olsen SK, Capili AD, Cisar JS, Lima CD, Tan DS. 2010. Designed semisynthetic protein inhibitors of $\mathrm{Ub} / \mathrm{Ubl} \mathrm{E} 1$ activating enzymes. J Am Chem Soc 132: 1748-1749. doi:10.1021/ ja9088549

Luo K, Zhang H, Wang L, Yuan J, Lou Z. 2012. Sumoylation of $M D C 1$ is important for proper DNA damage response. EMBO J 31: 3008-3019. doi:10.1038/emboj.2012.158

Luo K, Deng M, Li Y, Wu C, Xu Z, Yuan J, Lou Z. 2015. CDK-mediated RNF4 phosphorylation regulates homologous recombination in S-phase. Nucleic Acids Res 43: 5465-5475. doi:10 $.1093 / \mathrm{nar} / \mathrm{gkv} 434$

Makhnevych T, Ptak C, Lusk CP, Aitchison JD, Wozniak RW. 2007. The role of karyopherins in the regulated sumoylation of septins. J Cell Biol 177: 39-49. doi:10.1083/jcb.200608066

Morris JR, Garvin AJ. 2017. SUMO in the DNA double-stranded break response: similarities, differences, and cooperation with ubiquitin. J Mol Biol 429: 3376-3387. doi:10.1016/j.jmb.2017 .05 .012

Morris JR, Boutell C, Keppler M, Densham R, Weekes D, Alamshah A, Butler L, Galanty Y, Pangon L, Kiuchi T, et al. 2009. The SUMO modification pathway is involved in the BRCA1 response to genotoxic stress. Nature 462: 886-890. doi:10 $.1038 /$ nature 08593

Mukhopadhyay D, Dasso M. 2007. Modification in reverse: the SUMO proteases. Trends Biochem Sci 32: 286-295. doi:10 $.1016 /$ j.tibs.2007.05.002

Nishi R, Wijnhoven P, le Sage C, Tjeertes J, Galanty Y, Forment JV, Clague MJ, Urbé S, Jackson SP. 2014. Systematic characterization of deubiquitylating enzymes for roles in maintaining genome integrity. Nat Cell Biol 16: 1016-1026. doi:10.1038/ ncb3028

Nowsheen S, Aziz K, Aziz A, Deng M, Qin B, Luo K, Jeganathan KB, Zhang H, Liu T, Yu J, et al. 2018. L3MBTL2 orchestrates ubiquitin signalling by dictating the sequential recruitment of RNF8 and RNF168 after DNA damage. Nat Cell Biol 20: 455-464. doi:10.1038/s41556-018-0071-x

Odeh HM, Coyaud E, Raught B, Matunis MJ. 2018. The SUMOspecific isopeptidase SENP2 is targeted to intracellular membranes via a predicted $\mathrm{N}$-terminal amphipathic a-helix. Mol Biol Cell 29: 1878-1890. doi:10.1091/mbc.E17-07-0445

Ouyang KJ, Woo LL, Zhu J, Huo D, Matunis MJ, Ellis NA. 2009. SUMO modification regulates BLM and RAD51 interaction at damaged replication forks. PLOS Biol 7: e1000252. doi:10 .1371/journal.pbio.1000252

Panier S, Boulton SJ. 2014. Double-strand break repair: 53BP1 comes into focus. Nat Rev Mol Cell Biol 15: 7-18. doi:10 $.1038 / \mathrm{nrm} 3719$

Panse VG, Küster B, Gerstberger T, Hurt E. 2003. Unconventional tethering of Ulp1 to the transport channel of the nuclear pore complex by karyopherins. Nat Cell Biol 5: 21-27. doi:10.1038/ ncb893

Pfeiffer A, Luijsterburg MS, Acs K, Wiegant WW, Helfricht A, Herzog LK, Minoia M, Böttcher C, Salomons FA, van Attikum $\mathrm{H}$, et al. 2017. Ataxin-3 consolidates the MDC1-dependent DNA double-strand break response by counteracting the SUMO-targeted ubiquitin ligase RNF4. EMBO I 36: 10661083. doi:10.15252/embj.201695151

Plechanovova A, Jaffray EG, Tatham MH, Naismith JH, Hay RT. 2012. Structure of a RING E3 ligase and ubiquitin-loaded E2 primed for catalysis. Nature 489: 115-120. doi:10.1038/ nature 11376

Poulsen SL, Hansen RK, Wagner SA, van Cuijk L, van Belle GJ, Streicher W, Wikstrom M, Choudhary C, Houtsmuller AB, Marteijn JA, et al. 2013. RNF111/Arkadia is a SUMO-targeted ubiquitin ligase that facilitates the DNA damage response. I Cell Biol 201: 797-807. doi:10.1083/jcb.201212075

Psakhye I, Jentsch S. 2012. Protein group modification and synergy in the SUMO pathway as exemplified in DNA repair. Cell 151: 807-820. doi:10.1016/j.cell.2012.10.021

Qian J, Massion PP. 2008. Role of chromosome 3q amplification in lung cancer. J Thorac Oncol 3: 212-215. doi:10.1097/JTO .0b013e3181663544

Rojas-Fernandez A, Plechanovová A, Hattersley N, Jaffray E, Tatham MH, Hay RT. 2014. SUMO chain-induced dimerization activates RNF4. Mol Cell 53: 880-892. doi:10.1016/j .molcel.2014.02.031

Savic V, Yin B, Maas NL, Bredemeyer AL, Carpenter AC, Helmink BA, Yang-Iott KS, Sleckman BP, Bassing CH. 2009. Formation of dynamic $\gamma$-H2AX domains along broken DNA strands is distinctly regulated by ATM and MDC1 and dependent upon H2AX densities in chromatin. Mol Cell 34: 298310. doi:10.1016/j.molcel.2009.04.012

Seeler JS, Dejean A. 2017. SUMO and the robustness of cancer. Nat Rev Cancer 17: 184-197. doi:10.1038/nrc.2016.143

Shao G, Lilli DR, Patterson-Fortin J, Coleman KA, Morrissey DE, Greenberg RA. 2009. The Rap80-BRCC36 de-ubiquitinating enzyme complex antagonizes RNF8-Ubc13-dependent ubiquitination events at DNA double strand breaks. Proc Natl Acad Sci 106: 3166-3171. doi:10.1073/pnas.0807485106

Shima H, Suzuki H, Sun JY, Kono K, Shi L, Kinomura A, Horikoshi Y, Ikura T, Ikura M, Kanaar R, et al. 2013. Activation of the SUMO modification system is required for the accumulation of RAD51 at sites of DNA damage. J Cell Sci 126: 52845292. doi:10.1242/jcs.133744

Song J, Durrin LK, Wilkinson TA, Krontiris TG, Chen Y. 2004. Identification of a SUMO-binding motif that recognizes SUMO-modified proteins. Proc Natl Acad Sci 101: 1437314378. doi:10.1073/pnas.0403498101 
Stewart GS, Panier S, Townsend K, Al-Hakim AK, Kolas NK, Miller ES, Nakada S, Ylanko I, Olivarius S, Mendez M, et al. 2009. The RIDDLE syndrome protein mediates a ubiquitin-dependent signaling cascade at sites of DNA damage. Cell 136: 420-434. doi:10.1016/j.cell.2008.12.042

Stucki M, Clapperton JA, Mohammad D, Yaffe MB, Smerdon SJ, Jackson SP. 2005. MDC1 directly binds phosphorylated histone H2AX to regulate cellular responses to DNA double-strand breaks. Cell 123: 1213-1226. doi:10.1016/j.cell.2005.09.038

Tammsalu T, Matic I, Jaffray EG, Ibrahim AF, Tatham MH, Hay RT. 2014. Proteome-wide identification of SUMO2 modification sites. Sci Signal 7: rs2. doi:10.1126/scisignal.2005146

Tan M, Gong H, Wang J, Tao L, Xu D, Bao E, Liu Z, Qiu J. 2015. SENP2 regulates MMP13 expression in a bladder cancer cell line through SUMOylation of TBL1/TBLR1. Sci Rep 5: 13996. doi:10.1038/srep13996

Tatham MH, Geoffroy MC, Shen L, Plechanovova A, Hattersley N, Jaffray EG, Palvimo JJ, Hay RT. 2008. RNF4 is a polySUMO-specific E3 ubiquitin ligase required for arsenic-induced PML degradation. Nat Cell Biol 10: 538-546. doi:10 $.1038 /$ ncb1716

Thorslund T, Ripplinger A, Hoffmann S, Wild T, Uckelmann M, Villumsen B, Narita T, Sixma TK, Choudhary C, Bekker-Jensen $\mathrm{S}$, et al. 2015. Histone $\mathrm{H} 1$ couples initiation and amplification of ubiquitin signalling after DNA damage. Nature 527: 389-393. doi:10.1038/nature15401

Torrecilla I, Oehler J, Ramadan K. 2017. The role of ubiquitin-dependent segregase p97 (VCP or Cdc48) in chromatin dynamics after DNA double strand breaks. Philos Trans $R$ Soc Lond $B$ Biol Sci 372: 20160282. doi:10.1098/rstb.2016.0282
Uckelmann M, Sixma TK. 2017. Histone ubiquitination in the DNA damage response. DNA Repair (Amst) 56: 92-101. doi:10.1016/j.dnarep.2017.06.011

Vyas R, Kumar R, Clermont F, Helfricht A, Kalev P, Sotiropoulou P, Hendriks IA, Radaelli E, Hochepied T, Blanpain C, et al. 2013. RNF4 is required for DNA double-strand break repair in vivo. Cell Death Differ 20: 490-502. doi:10.1038/cdd.2012 .145

Yin YL, Seifert A, Chua JS, Maure JF, Golebiowski F, Hay RT. 2012. SUMO-targeted ubiquitin E3 ligase RNF4 is required for the response of human cells to DNA damage. Genes Dev 26: 1196-1208. doi:10.1101/gad.189274.112

Yu B, Swatkoski S, Holly A, Lee LC, Giroux V, Lee CS, Hsu D, Smith JL, Yuen G, Yue J, et al. 2015. Oncogenesis driven by the Ras/Raf pathway requires the SUMO E2 ligase Ubc9. Proc Natl Acad Sci 112: E1724-E1733. doi:10.1073/pnas .1415569112

Yurchenko V, Xue Z, Sadofsky MJ. 2006. SUMO modification of human XRCC4 regulates its localization and function in DNA double-strand break repair. Mol Cell Biol 26: 1786-1794. doi:10.1128/MCB.26.5.1786-1794.2006

Yurchenko V, Xue Z, Gama V, Matsuyama S, Sadofsky MJ. 2008. $\mathrm{Ku} 70$ is stabilized by increased cellular SUMO. Biochem Biophys Res Commun 366: 263-268. doi:10.1016/j.bbrc.2007.11 .136

Zhang H, Saitoh H, Matunis MJ. 2002. Enzymes of the SUMO modification pathway localize to filaments of the nuclear pore complex. Mol Cell Biol 22: 6498-6508. doi:10.1128/ MCB.22.18.6498-6508.2002 


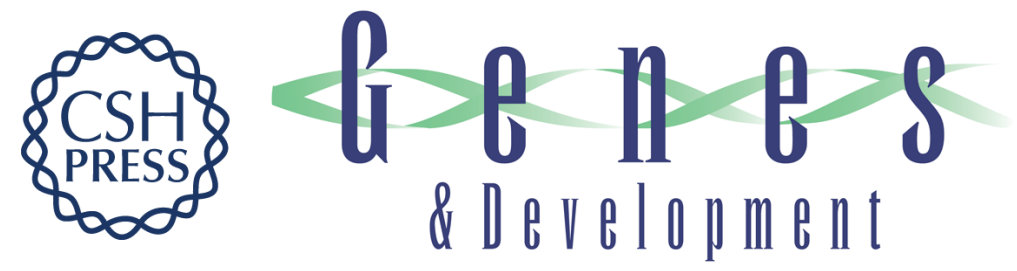

\section{The deSUMOylase SENP2 coordinates homologous recombination and nonhomologous end joining by independent mechanisms}

Alexander J. Garvin, Alexandra K. Walker, Ruth M. Densham, et al.

Genes Dev. 2019, 33: originally published online February 22, 2019

Access the most recent version at doi:10.1101/gad.321125.118

\section{Supplemental http://genesdev.cshlp.org/content/suppl/2019/02/22/gad.321125.118.DC1 \\ Material}

References This article cites 76 articles, 20 of which can be accessed free at: http://genesdev.cshlp.org/content/33/5-6/333.full.html\#ref-list-1

Creative This article, published in Genes \& Development, is available under a Creative Commons Commons License (Attribution-NonCommercial 4.0 International), as described at License http://creativecommons.org/licenses/by-nc/4.0/.

Email Alerting Receive free email alerts when new articles cite this article - sign up in the box at the top Service right corner of the article or click here.

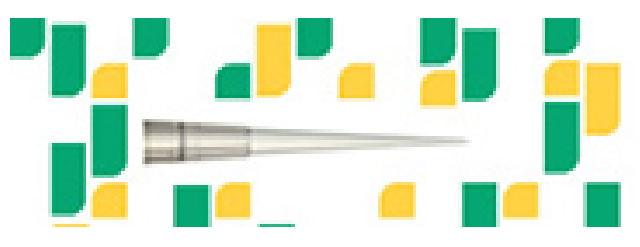

Focused on your science. 